\title{
Post common envelope binaries from SDSS
}

\section{The orbital period distribution $\star, \star \star$}

\author{
A. Nebot Gómez-Morán ${ }^{1,2}$, B. T. Gänsicke ${ }^{3}$, M. R. Schreiber ${ }^{4}$, A. Rebassa-Mansergas ${ }^{4}$, A. D. Schwope ${ }^{2}$, \\ J. Southworth ${ }^{5}$, A. Aungwerojwit ${ }^{6,7}$, M. Bothe ${ }^{2}$, P. J. Davis ${ }^{8}$, U. Kolb ${ }^{9}$, M. Müller ${ }^{2}$, C. Papadaki ${ }^{10}$, \\ S. Pyrzas ${ }^{3}$, A. Rabitz ${ }^{2}$, P. Rodríguez-Gil ${ }^{11,12,13}$, L. Schmidtobreick ${ }^{14}$, R. Schwarz ${ }^{2}$, C. Tappert ${ }^{4}$, \\ O. Toloza ${ }^{4}$, J. Vogel ${ }^{2}$, and M. Zorotovic ${ }^{4,15}$
}

1 Université de Strasbourg, CNRS, UMR 7550, Observatoire Astronomique de Strasbourg, 11 rue de l'Université, 67000 Strasbourg, France e-mail: ada.nebot@astro.unistra.fr

${ }^{2}$ Leibniz-Institut für Astrophysik Potsdam, An der Sternwarte 16, 14482 Potsdam, Germany

3 Department of Physics, University of Warwick, Coventry CV4 7AL, UK

4 Departamento de Física y Astronomía, Facultad de Ciencias, Universidad de Valparaíso, Valparaíso, Chile

5 Astrophysics Group, Keele University, Staffordshire, ST5 5BG, UK

6 Department of Physics, Faculty of Science, Naresuan University, 65000 Phitsanulok, Thailand

7 ThEP Centre, CHE, 328 Si Ayutthaya Road, 10400 Bangkok, Thailand

8 Institut d'Astronomie et d'Astrophysique, Université Libre de Bruxelles, CP226, Boulevard du Triomphe, 1050, Belgium

9 Open University, Dept. Physics \& Astronomy, Milton Keynes MK7 6BJ, UK

10 Institute of Astronomy \& Astrophysics, National Observatory of Athens, 15236 Athens, Greece

11 Instituto de Astrofísica de Canarias, vía Láctea, s/n, La Laguna, 38205 Tenerife, Spain

12 Departamento de Astrofísica, Universidad de La Laguna, Avda. Astrofísico Fco. Sánchez, sn, La Laguna, 38206 Tenerife, Spain

13 Isaac Newton Group of Telescopes, Apartado de correos 321, S/C de la Palma, 38700 Canary Islands, Spain

14 European Southern Observatory, Alonso de Córdova 3107, Vitacura, Santiago, Chile

15 Departamento de Astronomía y Astrofísica, Pontificia Universidad Católica, Vicuña Mackenna 4860, $782-0436$ Macul, Chile

Received 17 June 2011 / Accepted 20 September 2011

\section{ABSTRACT}

Context. The complexity of the common-envelope phase and of magnetic stellar wind braking currently limits our understanding of close binary evolution. Because of their intrinsically simple structure, observational population studies of white dwarf plus main sequence (WDMS) binaries can potentially test theoretical models and constrain their parameters.

Aims. The Sloan Digital Sky Survey (SDSS) has provided a large and homogeneously selected sample of WDMS binaries, which we characterise in terms of orbital and stellar parameters.

Methods. We have obtained radial velocity information for 385 WDMS binaries from follow-up spectroscopy and for an additional 861 systems from the SDSS subspectra. Radial velocity variations identify 191 of these WDMS binaries as post common-envelope binaries (PCEBs). Orbital periods of 58 PCEBs were subsequently measured, predominantly from time-resolved spectroscopy, bringing the total number of SDSS PCEBs with orbital parameters to 79. Observational biases inherent to this PCEB sample were evaluated through extensive Monte Carlo simulations.

Results. We find that 21-24\% of all SDSS WDMS binaries have undergone common-envelope evolution, which is in good agreement with published binary population models and high-resolution HST imaging of WDMS binaries unresolved from the ground. The bias-corrected orbital period distribution of PCEBs ranges from $1.9 \mathrm{~h}$ to $4.3 \mathrm{~d}$ and approximately follows a normal distribution in $\log \left(P_{\text {orb }}\right)$, peaking at $\sim 10.3 \mathrm{~h}$. There is no observational evidence for a significant population of PCEBs with periods in the range of days to weeks.

Conclusions. The large and homogeneous sample of SDSS WDMS binaries provides the means to test fundamental predictions of binary population models, hence to observationally constrain the evolution of all close compact binaries.

Key words. binaries: close - binaries: spectroscopic - white dwarfs - stars: low-mass

\section{Introduction}

It is well known that all types of close compact binaries, including X-ray binaries, cataclysmic variables, and

* Figures 3-6, Tables 1,5 and Appendices are only available in electronic form at http://www . aanda.org

$\star \star$ Full Tables 3, 4, and 6 are only available at the CDS via anonymous ftp to cdsarc.u-strasbg. fr $(130.79 .128 .5)$ or via http://cdsarc.u-strasbg.fr/viz-bin/qcat?J/A+A/536/A43 double degenerates, form through common-envelope evolution (Paczyński 1976). This phase is required to explain the observed small binary separations in compact binaries. The main concept is that, for a suitable range of orbital separations, the massive star (i.e. the primary) will fill its Roche lobe as it evolves up the giant (or asymptotic giant) branch, and will then in most cases become susceptible to dynamically unstable mass transfer. The transferred material cannot cool as fast as it is being transferred, and the core of the primary star and the companion 
star (also referred to as secondary star) are both engulfed by the envelope of the former. The envelope does not co-rotate with the binary, thereby creating drag forces that transport orbital angular momentum and energy from the binary to the common envelope, leading to a shrinkage of the orbit (Ricker \& Taam 2008). Eventually, the common-envelope is ejected, leaving behind a post common-envelope binary (PCEB).

Although the basic concept of the common-envelope phase has been outlined 30 years ago, it is still the most poorly understood phase of compact binary evolution. While we have learnt that this phase must be very short $\left(\leq 10^{3} \mathrm{yrs}\right)$ (Hjellming \& Taam 1991; Webbink 2008), current hydrodynamical simulations of common-envelope evolution are still unable to unambiguously link the initial parameters of the binary with the outcome of the common-envelope, and numerically too expensive to be run using a large number of initial binary parameters (Ricker \& Taam 2008). As a result, theoretical binary population synthesis studies (BPS) usually adopt a simple parametrisation: a certain fraction $\left(\alpha_{\mathrm{CE}}\right)$ of the binary's binding energy that is released in the spiralling-in process is used to unbind the common envelope (Webbink 1984). However, Nelemans et al. (2000) and Nelemans \& Tout (2005) find difficulties with this scaled energy relation when trying to reproduce the evolution of double white dwarf binaries, and propose a prescription based on angular momentum conservation. A fundamental consequence of this approach is that clear observational constraints on these parameters are needed to meaningfully predict the Galactic population of compact binaries.

PCEBs ${ }^{1}$ composed of a white dwarf and a main-sequence (WDMS) companion represent the most promising population for deriving such observational constraints, because i) they are intrinsically the most abundant population of PCEBs, so easily accessible to intense studies using the current suite of 2-8 m telescopes, ii) they underwent only one common-envelope phase, iii) are not altered by ongoing accretion, and iv) their stellar components are structurally simple. In general, WDMS binaries comprise two fundamentally different types of systems: wide binaries that evolved like single stars, i.e. without interaction, and PCEBs. The major shortcoming of using PCEBs for studying compact binary evolution has been how few there are. The comprehensive study of Schreiber \& Gänsicke (2003) contained only 30 systems with accurate binary parameters, and it highlighted that the sample of PCEBs known at that time was not only small but also heavily biased towards hot white dwarfs and late secondary star spectral types. This bias was identified by Schreiber \& Gänsicke (2003) as a natural consequence of the fact that most of these PCEBs were discovered in blue-excess surveys.

Over the past few years, the Sloan Digital Sky Survey (SDSS, York et al. 2000; Abazajian et al. 2009; Yanny et al. 2009) has totally changed this situation. Thanks to its broad ugriz colour space, and massive spectroscopic follow-up, SDSS has identified over 1900 WDMS binaries (Smolčić et al. 2004; Silvestri et al. 2006, 2007; Schreiber et al. 2007; Heller et al. 2009; Rebassa-Mansergas et al. 2007; Rebassa-Mansergas et al. 2010). We have initiated an intensive follow-up study of the SDSS WDMS binaries, with the ultimate aim to substantially improve our understanding of compact binary evolution. First results on individual systems and small subsets of this sample have been published (Schreiber et al. 2008; Rebassa-Mansergas et al. 2008; Nebot Gómez-Morán et al. 2009; Pyrzas et al. 2009;

\footnotetext{
1 For the remainder of the paper, we use $P C E B$ as a synonym for a short-period WDMS binary that underwent a common-envelope phase.
}

Schwope et al. 2009), as well as several studies that analyse the total sample in the context of close binary evolution (Schreiber et al. 2010; Zorotovic et al. 2010; Rebassa-Mansergas et al. 2011b). Here we present one of the fundamental results of this project: the orbital period distribution of 79 SDSS WDMS binaries, which we discuss in the context of current theories of compact binary evolution.

\section{Overall strategy and input sample}

Our aim of characterising a large and homogeneous sample of PCEBs requires a multi-stage approach. The first step is to identify all WDMS binaries within the SDSS spectroscopic data release. The latest catalogues of WDMS binaries from SDSS list 1903 WDMS systems, 1600 from Rebassa-Mansergas et al. (2010) based on the SDSS Data Release (DR) 6, plus another 300 that have been discovered by us (Schreiber et al. 2007; Rebassa-Mansergas et al. 2011a) as part of the Sloan Extension for Galactic Understanding and Exploration (SEGUE, Yanny et al. 2009). These catalogues include white dwarf temperatures and masses, as well as the spectral types of the companion stars. In brief, stellar parameters are derived from fitting the SDSS spectrum to template spectra made of white dwarf and $\mathrm{dM}$ templates. The spectral type of the secondary star is derived from the best fit, and the mass is calculated using a spectral type-mass relation (see Rebassa-Mansergas et al. 2007, for details). The mass and the temperature of the white dwarf are derived from fitting both lines and continuum (after the contribution from the companion has been subtracted) to stellar models from Koester et al. (2005).

The second step is to identify the PCEBs among the entire sample of WDMS binaries. The comprehensive population synthesis by Willems \& Kolb (2004) suggests that $\sim 75 \%$ of all Galactic WDMS binaries are wide systems and that the vast majority of the remaining $\$ 25 \%$ underwent a common-envelope phase, hence several hundred PCEBs are to be expected among the SDSS WDMS sample. A small fraction of WDMS binaries appear as blended white dwarf plus M-dwarf pairs in the SDSS imaging, and were flagged as wide systems by RebassaMansergas et al. (2010). However, even those WDMS binaries that are genuinely unresolved on the SDSS images can still have orbital separations of many tens of astronomical units. The key to identifing the close WDMS binaries is therefore to carry out a radial velocity survey of the spatially unresolved SDSS WDMS binaries, obtaining at least two spectra separated by at least one night. In a final step, intense follow-up spectroscopy of the PCEBs, i.e. WDMS binaries found to be radial velocity variable, is needed to measure their orbital periods.

\section{Observations, reduction, and radial velocity measurements}

We obtained spectroscopic follow-up observations for 385 systems (Sect. 3.1), and used exclusively SDSS spectroscopic data for another 861 systems, bringing the number of SDSS WDMS binaries with at least two reliable radial velocity measurements to 1246 . In addition, we obtained photometric timeseries for seven systems (Sect. 3.3). A log of the observations is given in Table 1, listing the name of the object, the ugriz magnitudes, the telescopes, the number of spectra taken, and the duration of the observations. 
Table 2. Observational technical details.

\begin{tabular}{|c|c|c|c|c|c|}
\hline & & Spectroscopy & & & \\
\hline Tel./Inst. & $\begin{array}{c}\text { Slit } \\
\text { width }\left[{ }^{\prime \prime}\right]\end{array}$ & $\begin{array}{c}\text { Spect. } \\
\text { range }[\AA]\end{array}$ & $\begin{array}{l}\text { Spect. } \\
\text { res. }[\AA]]\end{array}$ & $\begin{array}{c}\left\langle\sigma_{\mathrm{RV}}\right\rangle \\
{\left[\mathrm{km} \mathrm{s}^{-1}\right]}\end{array}$ & $\begin{array}{l}\text { \# of } \\
\text { RVs }\end{array}$ \\
\hline VLT/FORS2 & 1 & $7830-9570$ & 2.2 & 7.1 & 334 \\
\hline Gemini-N/GMOS & 1 & $7770-8470$ & 3.5 & 7.1 & 124 \\
\hline Gemini-S/GMOS & 1 & $7770-8470$ & 4.1 & 8.6 & 26 \\
\hline Clay ${ }^{\ddagger} / \mathrm{LDSS} 3$ & 0.75 & $5800-9980$ & 4.8 & 10.7 & 302 \\
\hline Baade & 0.75 & $7700-8560$ & 1.5 & 11.0 & 187 \\
\hline WHT/ISIS & 1 & $7600-9000$ & 1.6 & 9.2 & 524 \\
\hline NTT/EMMI & 1 & $7770-8830$ & 2.8 & 11.4 & 284 \\
\hline NTT/EFOSC2 & 1 & $7000-8600$ & 6.5 & 19.5 & 94 \\
\hline CA3.5/TWIN & 1.5 & $7500-8500$ & 1.6 & 11.6 & 443 \\
\hline CA3.5/MOSCA & 1.5 & $5400-9000$ & 7 & 17.0 & 12 \\
\hline SDSS & $3^{\dagger}$ & $3900-9100$ & 4 & 19.1 & 5171 \\
\hline Tel./Inst. & & $\begin{array}{c}\text { Photometry } \\
\text { Resolution ["/pix.] }\end{array}$ & & \# of images & \\
\hline IAC80/CAMELOT & & 0.6 & & 397 & \\
\hline DuPont/CCD & & 0.259 & & 337 & \\
\hline CA2.2/CAFOS & & 0.53 & & 1083 & \\
\hline
\end{tabular}

Notes. ${ }^{(\dagger)}$ SDSS uses a fibre-fed spectrograph. ${ }^{(\ddagger)}$ The two Magellan $6.5 \mathrm{~m}$ telescopes.

\subsection{Spectroscopy}

Throughout the period January 2006 to July 2010, we used the $3.5 \mathrm{~m}$ telescope at Calar Alto (CA), the $3.5 \mathrm{New}$ Technology Telescope (NTT), the $4.2 \mathrm{~m}$ William Herschel Telescope (WHT), the two $6.5 \mathrm{~m}$ Magellan telescopes (Baade/Clay), the two $8 \mathrm{~m}$ Gemini telescopes (Gemini-N/S), and the $8 \mathrm{~m}$ Very Large Telescope (VLT) to obtain spectroscopy of 385 SDSS WDMS binaries. Details on the instrumentation and setup are given in Table 2.

The data obtained at Calar Alto were reduced within MIDAS $^{2}$, and the spectra were extracted using the optimal algorithm (Horne 1986). The data from all other telescopes was reduced using the STARLINK ${ }^{3}$ packages FIGARO and KAPPA, and spectra were optimally extracted using PAMELA (Marsh 1989) and wavelength-calibrated within MOLLY 4 . For all the spectra, the wavelength calibration was checked and, if necessary, corrected for telescope/instrument flexure using the night sky lines. The spectra were flux-calibrated and corrected for telluric absorption using observations of the stars $\mathrm{BD}+28^{\circ} 4211, \mathrm{BD}+33+{ }^{\circ} 2642, \mathrm{BD}+25^{\circ} 4655$, Feige 66 , Feige 110, GD 108, and LTT 3218. For additional details on the reduction and calibration of the data, see Schreiber et al. (2008); Rebassa-Mansergas et al. (2008) and Nebot Gómez-Morán et al. (2009).

In addition to our own follow-up spectroscopy, we made use of the spectroscopic data from SDSS. Every SDSS spectrum is the average of (typically) three $15 \mathrm{~min}$ exposures. These subspectra have been publicly available since DR6 (AdelmanMcCarthy et al. 2008). As shown by Rebassa-Mansergas et al. (2008) and Schwope et al. (2009) it is possible to use many of these subspectra to measure radial velocities and identify short-period WDMS binaries. We retrieved the subspectra for all 1903 systems in our input catalogue. Discarding spectra with a signal-to-noise ratio that is too low, we were able to measure at least one radial velocity for 1147 WDMS binaries, and we measured a total of 5171 radial velocities using the SDSS data. The wavelength calibration of the SDSS spectra can be accurate

\footnotetext{
2 http://www.eso.org/sci/software/esomidas

3 starlink.jach.hawaii.edu/starlink

${ }^{4}$ www.warwick.ac.uk/go/trmarsh/software
}

to $\simeq 2 \mathrm{~km} \mathrm{~s}^{-1}$ in dark nights, while in bright nights there can be shifts up to $20 \mathrm{~km} \mathrm{~s}^{-1}$. A systematic error of $7 \mathrm{~km} \mathrm{~s}^{-1}$ is reported by Stoughton et al. (2002) and Yanny et al. (2009). Although the SEGUE spectroscopic data has been corrected for that offset, this shift has only been applied to the combined spectra and not the individual subspectra. We take this into account by adding in quadrature $10 \mathrm{~km} \mathrm{~s}^{-1}$ to the statistical errors on the radial velocities measured from Gaussian fits (see Sect. 3.2).

\subsection{Radial velocity measurements}

We measured the radial velocities of the companion stars by fitting a second-order polynomial plus two Gaussians to the observed 8183/8194 $\AA \mathrm{NaI}$ absorption doublet. The separation of the two Gaussians was fixed to the laboratory value of the $\mathrm{Na}$ I doublet. We also measured the radial velocities from the $\mathrm{H} \alpha$ emission line (where present) by fitting a second-order polynomial to the underlying continuum, plus a Gaussian for the emission line (see Rebassa-Mansergas et al. 2007, 2008, for further details on the method).

\subsection{Photometric follow-up observations}

We carried out time-series photometry for seven WDMS binaries to probe for orbital variability (Table 1) where the available spectroscopy suggested a short orbital period. These data were obtained between September 2006 and August 2009 using the $2.5 \mathrm{~m}$ DuPont telescope, the $2.2 \mathrm{~m}$ telescope at Calar Alto, and the $0.8 \mathrm{~m}$ IAC 80 telescope. All observations were carried out with red filters (Bessel $R$, Sloan $r$ or $i$ ) to maximise the sensitivity to changes in the brightness of the secondary star (ellipsoidal modulation and/or reflection effect). The IAC80 data were reduced with the standard packages in $\mathrm{IRAF}^{5}$. The DuPont and Calar Alto data were de-biased and flat-fielded in a standard fashion within MIDAS, and instrumental magnitudes were derived using SExtractor (Bertin \& Arnouts 1996). For a full description of the photometric reduction pipeline see Gänsicke et al. (2004).

The results of the photometric observations are discussed in Sects. 4.2.1 and 4.2.3, and details on individual systems are given in Appendix A.

\section{Observational results}

\subsection{Post common-envelope binary identification}

Our observational program started back in 2005. The original strategy for identification of PCEBs was to take two to three spectra for each WDMS binary at random times, with the only constraint being that they should be separated by at least one night. Any system displaying significant radial velocity variations was then flagged for intense follow-up spectroscopy to measure its orbital period.

With the wealth of SDSS increasing with each data release, our strategy has evolved. Within DR5, Rebassa-Mansergas et al. (2007) found that 100 WDMS had multiple SDSS spectra, and identified some of the PCEBs among them. As mentioned in Sect. 3.1 each SDSS spectrum is typically the average of three individual exposures. Occasionally, the subspectra were

\footnotetext{
5 IRAF is distributed by the National Optical Astronomy Observatory, which is operated by the Association of Universities for Research in Astronomy, Inc., under contract with the National Science Foundation, http://iraf. noao. edu
} 

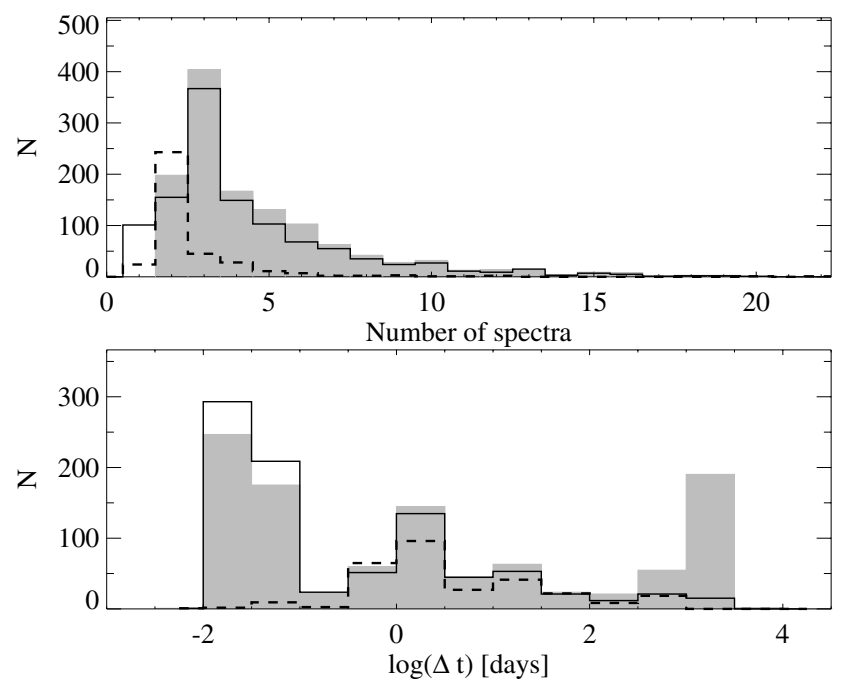

Fig. 1. Upper panel: frequency of the number of spectra taken per system from our spectroscopic campaign (dashed), from the SDSS (solid) and total combined number of spectra (grey). Bottom panel: distribution of time span between the first and the last spectra taken for identification of close and wide binaries. See text for a detailed explanation.

obtained on different nights, and for a small number of objects more than three subspectra are available. These subspectra were made publicly available from DR6 onwards, and we made use of them to identify PCEBs. For $\sim 100$ systems, the signal-to-noise ratio of the subspectra was too low for an accurate radial velocity measurement. In these cases, we combined the radial velocity determined from the co-added SDSS spectra with our own measurements. For those WDMS binaries that have SDSS data obtained on different nights, we usually took only one additional spectrum to have an independent measurement.

Figure 1 shows the distribution of the number of spectra per system used to separate PCEBs and wide binary candidates (upper panel), and the time elapsed between the first and last spectrum for a given object (bottom panel), for the SDSS data, our follow-up data, and the combined data. Most of the WDMS binaries have 3 SDSS subspectra which were taken in the same night, and are therefore sensitive only to short orbital periods. A second peak around a few days indicates SDSS repeat observations of the same spectroscopic plate. Our own spectroscopy was typically carried out with a separation of $\sim 1-3$ days in visitor-mode observing runs, with a long tail coming from service-mode observations on the VLT and on Gemini. Note that the combined distributions are not the direct sum of the other two, because there are systems that were observed only once by us but had several SDSS subspectra, or the other way round.

For the identification of radial-velocity variable stars, i.e. PCEBs, we analysed the radial velocities measured from the $\mathrm{NaI}$ doublet and the $\mathrm{H} \alpha$ emission line separately, since these two velocities can differ by more than the statistical errors (Rebassa-Mansergas et al. 2007), e.g. if $\mathrm{H} \alpha$ originates predominantly in the inner hemisphere of the companion. We followed the approach of Maxted et al. (2000) to evaluate the significance of the measured radial velocity differences. For each system we calculated the $\chi^{2}$ statistic with respect to the mean radial velocity, which represents the best fit for a non-variable system. For the hypothesis of a constant radial velocity, the probability $Q$ of obtaining a given $\chi^{2}$ is high when radial velocity variations are dominated by random noise, and low for intrinsically radial-velocity variable systems. The probability of

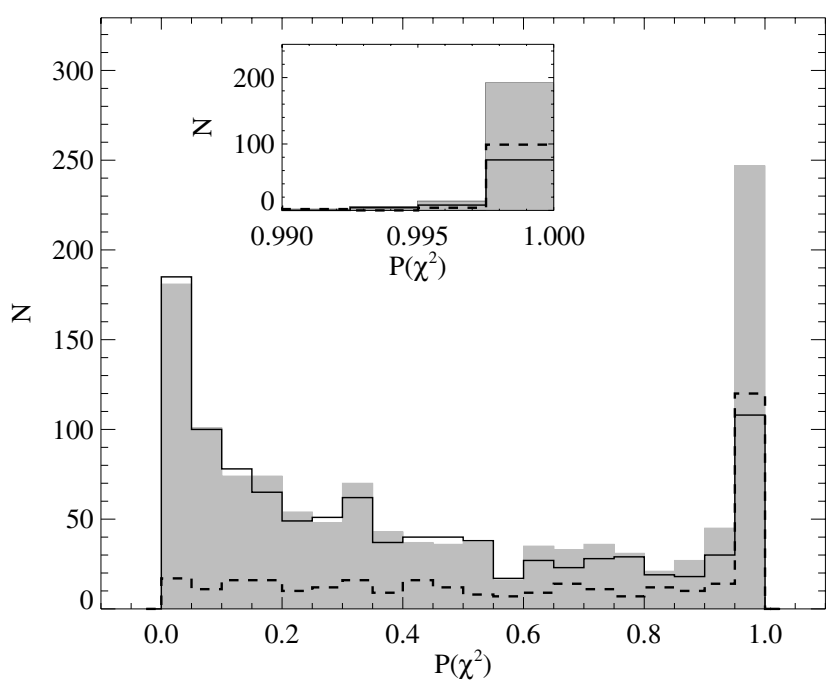

Fig. 2. Distribution of the probability of measuring strong radial velocity variations among the WDMS binaries in the sample. Those above a $3 \sigma\left(P\left(\chi^{2}\right)>99.73\right)$ threshold are considered to be PCEBs. Line coding as in Fig. 1. A zoom in the region between 0.995 and 1 is shown in the box, with a smaller binsize. For a detailed explanation see text.

Table 3. Radial velocities used for the identification of the binaries.

\begin{tabular}{lcccc}
\hline \hline $\begin{array}{l}\text { Name } \\
\text { SDSSJ }\end{array}$ & RV & err & hjd & Teles. \\
\hline $\left.\mathrm{km} \mathrm{s}^{-1}\right]$ & {$\left[\mathrm{km} \mathrm{s}^{-1}\right]$} & {$[$ days $]$} & \\
\hline $002749.99-001023.3$ & 13.2 & 15.3 & 2452262.628080 & SDSS \\
$002749.99-001023.3$ & 11.0 & 17.3 & 2452262.640324 & SDSS \\
$002749.99-001023.3$ & 7.0 & 17.0 & 2452262.652580 & SDSS \\
$002749.99-001023.3$ & 8.4 & 10.5 & 2454347.439589 & WHT \\
$002749.99-001023.3$ & 15.8 & 6.7 & 2454348.551886 & WHT \\
$002801.68+002137.7$ & -25.6 & 22.4 & 2452262.628105 & SDSS \\
$002801.68+002137.7$ & 1.8 & 21.0 & 2452262.640349 & SDSS \\
$002801.68+002137.7$ & 0.0 & 33.6 & 2452262.652605 & SDSS \\
$002801.68+002137.7$ & -4.8 & 5.0 & 2454381.694818 & VLT \\
$002801.68+002137.7$ & -3.3 & 5.0 & 2454382.804165 & VLT \\
$002950.16+003225.8$ & 18.3 & 21.0 & 2452262.628153 & SDSS \\
$002950.16+003225.8$ & 6.2 & 17.8 & 2452262.640397 & SDSS \\
$002950.16+003225.8$ & 31.5 & 27.4 & 2452262.652652 & SDSS \\
$002921.27-002032.8$ & -62.6 & 28.0 & 2452262.628107 & SDSS \\
$002921.27-002032.8$ & -25.6 & 17.0 & 2452262.640351 & SDSS \\
$002921.27-002032.8$ & -22.3 & 18.7 & 2452262.652607 & SDSS \\
$003221.86+073934.4$ & -326.6 & 8.3 & 2454681.908960 & Gemini-S \\
$003221.86+073934.4$ & -27.9 & 5.9 & 2454687.871683 & Gemini-S \\
$003336.49+004151.3$ & 67.8 & 17.2 & 2452930.850863 & SDSS \\
$003336.49+004151.3$ & 90.9 & 16.4 & 2452932.782491 & SDSS \\
\hline
\end{tabular}

a system showing high radial velocity variations is thus given by $P\left(\chi^{2}\right)=1-Q$. We consider strong PCEB candidates to be those systems showing radial velocity variations at a $>3 \sigma$ significance, or $P\left(\chi^{2}\right)>99.73 \%$. Radial velocities, errors, time of the observations, and the telescope used for the identification of the system are available via the CDS, and an excerpt is presented in Table 3. The number of spectra used for each system, time span covered by these spectra, and the probabilities $P\left(\chi^{2}\right)$ are available via the CDS, but see Table 4 for an extract.

The distribution of $P\left(\chi^{2}\right)$ is shown in Fig. 2, again for the SDSS data, our follow-up data, and the combined data. The combined probability distribution presents a pronounced peak at $P\left(\chi^{2}\right)=1$, corresponding to systems exhibiting a statistically significant radial velocity variation, i.e. PCEBs, and a less pronounced one close to $P\left(\chi^{2}\right)=0$, corresponding mainly to very wide systems, and a small contribution of PCEBs with very low 
A. Nebot Gómez-Morán et al.: The orbital period distribution of SDSS PCEBs. XII.

Table 4. Identification statistics.

\begin{tabular}{lcccc}
\hline \hline Name & $N$ & $N_{\text {obs }}$ & $\Delta t$ [days] & Prob. \\
\hline SDSSJ 000504.91+243409.6 & 10 & 3 & 294.821 & 0.74189 \\
SDSSJ 000531.09-054343.2 & 6 & 1 & 427.720 & 1.00000 \\
SDSSJ 000935.50+243251.2 & 16 & 9 & 377.809 & 0.97822 \\
SDSSJ 001749.24-000955.3 & 2 & 2 & 3.935 & 1.00000 \\
SDSSJ 002620.41+144409.5 & 3 & 0 & 0.029 & 0.32658 \\
SDSSJ 003221.86+073934.4 & 2 & 2 & 5.963 & 1.00000 \\
SDSSJ 004020.07+154156.3 & 4 & 0 & 66.847 & 0.99943 \\
SDSSJ 005008.21-000359.0 & 4 & 0 & 24.005 & 0.07446 \\
SDSSJ 005245.11-005337.2 & 6 & 5 & 2127.066 & 0.99999 \\
\hline
\end{tabular}

Notes. Name of the system, total number of spectra used for identification, number of spectra from own spectroscopic observations, time span between first and last observations and probability of measuring large radial velocity variations.

inclinations resulting in projected radial velocities that are too low to be picked up by the spectroscopic data. In total we found 191 PCEBs among the 1246 SDSS WDMS binaries for which at least two radial velocity measurements are available. These numbers can be used to set a strict lower limit on the fraction of PCEBs; see Sect. 6.1 for a more detailed analysis. For clarity, as for Fig. 1, we mention that the combined distribution of $P\left(\chi^{2}\right)$ is not the direct sum of the individual sets and illustrate this with an example: SDSS $0848+0058$ has three SDSS subspectra taken within $1.4 \mathrm{~h}$, and we took two spectra separated by five nights. We obtained $P\left(\chi^{2}\right)=0.4573$ and $P\left(\chi^{2}\right)=0.4014$ respectively. Combining the data we have five spectra, separated by seven years, and obtain $P\left(\chi^{2}\right)=0.7140$.

It is clear that any radial velocity survey will be subject to observational biases related to the spectral resolution employed, the number of spectra obtained for each object, and the temporal spacing of these spectra. These biases are evaluated in Sect. 5.2. They are also responsible for the differences between the probability distribution from the SDSS data and from our own followup observations, which are discussed in Sect. 5.

\subsection{Orbital period measurements}

As mentioned above, 191 WDMS binaries exhibited significant radial velocity variations and were flagged for detailed follow-up observations. Fifteen orbital periods have already been published by Pyrzas et al. (2011); Schreiber et al. (2008); Rebassa-Mansergas et al. (2008); Nebot Gómez-Morán et al. (2009), and Pyrzas et al. (2009). Here we report period determinations for 58 additional systems, four of which were measured from follow-up photometry, the rest from follow-up spectroscopy.

\subsubsection{Orbital periods from photometric observations}

Photometric variability in PCEBs can be caused by four different mechanisms: i) ellipsoidal modulation due to the distorted shape of the secondary star, ii) reflection effect on the surface of the secondary star due to irradiation from the hot primary, iii) eclipses, and iv) rotational modulation due to starspots (BY Dra phenomenon). The first three types of variability are, by definition, modulated on the orbital period. For most PCEBs, variability due to star spots will also be modulated on the orbital period, as the companion will by tidally synchronised. However, for wide binaries, star spots on the companion star may mimic the variability of a short-period PCEB. Four out of the seven WDMS binaries for which we obtained time-series photometry (Table 1) show ellipsoidal modulation, and we determined their orbital periods by fitting sine curves to the observations.
Table 6. Radial velocities used for measuring the orbital periods.

\begin{tabular}{lcccc}
\hline \hline $\begin{array}{l}\text { Name } \\
\text { SDSSJ }\end{array}$ & $\begin{array}{c}\text { RV } \\
{\left[\mathrm{km} \mathrm{s}^{-1}\right]}\end{array}$ & $\begin{array}{c}\text { err } \\
{\left[\mathrm{km} \mathrm{s}^{-1}\right]}\end{array}$ & $\begin{array}{c}\text { hjd } \\
\text { [days] }\end{array}$ & Teles. \\
\hline $015225.38-005808.5$ & 49.80 & 10.50 & 2454378.596674 & NTT \\
$015225.38-005808.5$ & 111.70 & 10.50 & 2454378.604076 & NTT \\
$015225.38-005808.5$ & 54.20 & 8.80 & 2454378.689058 & NTT \\
$015225.38-005808.5$ & -76.60 & 10.60 & 2454378.734465 & NTT \\
$015225.38-005808.5$ & -128.60 & 9.80 & 2454378.741869 & NTT \\
$015225.38-005808.5$ & -153.10 & 9.40 & 2454378.749270 & NTT \\
$015225.38-005808.5$ & 85.70 & 9.30 & 2454378.806674 & NTT \\
$015225.38-005808.5$ & -105.90 & 10.60 & 2454378.828943 & NTT \\
$015225.38-005808.5$ & -15.00 & 10.40 & 2454378.859215 & NTT \\
$015225.38-005808.5$ & 99.60 & 11.40 & 2454379.588580 & NTT \\
$015225.38-005808.5$ & -36.60 & 12.70 & 2454379.628128 & NTT \\
$015225.38-005808.5$ & -38.80 & 9.30 & 2454379.716397 & NTT \\
$015225.38-005808.5$ & -106.20 & 10.60 & 2454379.723800 & NTT \\
\hline
\end{tabular}

Periodograms and phase folded light curves are presented and discussed in more detail in Appendix A.

\subsubsection{Orbital periods from spectroscopic observations}

We obtained further spectroscopy for 68 of the 191 PCEBs identified by our radial velocity survey (Table 1 ). A period search was performed by computing periodograms using Schwarzenberg-Czerny's (1996) ORT/TSA method within MIDAS, which fits periodic orthogonal polynomials to the observations, combined with an analysis of variance statistic. In a second step, we fitted sine curves of the form

$v_{r}=\gamma_{\mathrm{sec}}+K_{\mathrm{sec}} \sin \left[\frac{2 \pi\left(t-t_{0}\right)}{P_{\mathrm{orb}}}\right]$

to the radial velocity data, with $\gamma_{\mathrm{sec}}$ the systemic velocity of the secondary star, $t_{0}$ the zero point defined by the inferior conjunction of the secondary star, and $K_{\mathrm{sec}}$ the radial velocity amplitude of the secondary star. Initial values for $P_{\text {orb }}$ were selected from the highest peaks in the periodograms. We adopted the values of the best-fit solution as orbital period, which always coincided with the highest peak in the periodogram. We could measure orbital periods for 58 of the 68 PCEBs that were spectroscopically followed up. For the remaining ten systems the radial velocity data was insufficient to unambiguously identify the true orbital periods. The radial velocity variations measured for these ten systems range from $50 \mathrm{~km} \mathrm{~s}^{-1}$ to $490 \mathrm{~km} \mathrm{~s}^{-1}$ so there is no a priori reason to think that their orbital period distribution differs significantly from the one drawn from the rest of the population of PCEBs.

The 58 orbital periods range from 1.97 to $104.56 \mathrm{~h}(0.08$ to 4.35 days) (Table 5). The spectra of these PCEBs, and their phase-folded radial velocity curves are shown in Figs. 3, and 5 and Figs. 4, and 6, respectively, and their stellar and binary parameters given in Table 5, which also lists $K_{\mathrm{sec}}$ and $\gamma_{\mathrm{sec}}$. The orbital period distribution of the SDSS PCEBs is discussed in more detail in Sect. 5. Radial velocities, errors, time of the observations, and the telescope used for measuring the orbital period of each system are available via the CDS, but see Table 6 for an extract.

\subsubsection{Orbital inclinations and candidate eclipsing systems}

We estimated the orbital inclinations and binary separations of those PCEBs for which masses of the white dwarf and the companion star (Sect. 2), $K_{2}$ and $P_{\text {orb }}$ were available using Kepler's 


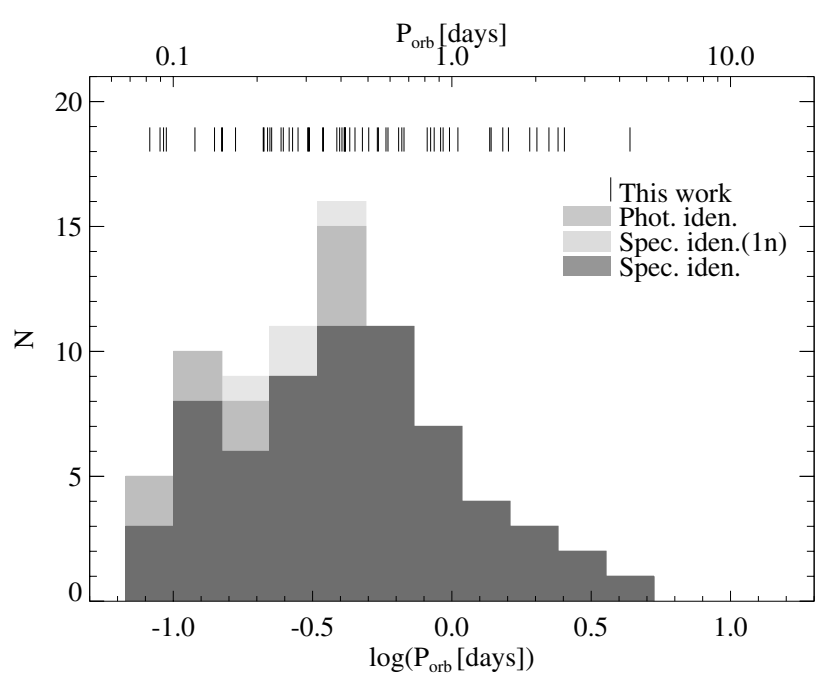

Fig. 7. Orbital period distribution of the 79 SDSS PCEBs characterised to date. Systems identified as PCEBs based on spectroscopic observations are shown in light grey if the spectra were taken during the same night and dark grey otherwise. In medium grey we show those systems that were identified as PCEBs based on photometry. The 58 systems added by this paper to the orbital period distribution are indicated with vertical lines.

third law, and propagated the errors in all quantities accordingly. We further calculated $K_{\mathrm{WD}}$, as well as the Roche-lobe size of the companion star, $R_{\text {lob }}$ using Eggleton's (1983) approximation. Finally, with the empirical radius-spectral type relation of Rebassa-Mansergas et al. (2007), we estimated the Roche-lobe filling factors of the secondary stars. All this ancillary information is given in Table 5. In case the effective temperature of the primary is lower than $12000 \mathrm{~K}$, the derived spectroscopic mass is inherently uncertain (Koester et al. 2009; Tremblay et al. 2010) and the derived parameters should be used with caution.

For six systems, the estimated orbital inclinations are close to 90 degrees, making them good candidates for being eclipsing binaries: SDSS 0238-0005, SDSS 1348+1834, SDSS 1434+5335, SDSS 1506-0120, SDSS 2132+0031, and SDSS 2318-0935. Eclipsing systems offer the best opportunity to derive accurate fundamental stellar parameters, i.e. masses and radii, and we have begun to exploit the potential offered by the eclipsing PCEBs (Steinfadt et al. 2008; Nebot Gómez-Morán et al. 2009; Pyrzas et al. 2009). SDSS 1348+1834, aka CSS 21357, was recently identified in the Catalina Real Time Transient Survey (Drake et al. 2009) as an eclipsing PCEB (Drake et al. 2010), confirming the predictive power of our estimates.

\subsubsection{Observed orbital period distribution}

We have measured here the orbital periods of 58 PCEBs. Together with the 15 periods already published by our group (Pyrzas et al. 2011; Rebassa-Mansergas et al. 2008; Schreiber et al. 2008; Pyrzas et al. 2009; Nebot Gómez-Morán et al. 2009), two more by Drake et al. (2010), and four additional PCEBs that are subject to forthcoming individual studies (Pyrzas et al., in prep.; Parsons et al., in prep.), the total number of PCEBs from SDSS with orbital period measurements stands at 79, which is more than double the corresponding number of pre-SDSS PCEBs. The orbital period distribution of the SDSS PCEBs is shown in Fig. 7 on a logarithmic scale. The 58 periods determined in this paper are indicated by vertical lines. Taking this observed period distribution at face value, PCEBs are found to have orbital periods ranging from $1.97 \mathrm{~h}$ to $4.35 \mathrm{~d}$, and they approximately follow a normal distribution in $\log \left(P_{\text {orb }}\right)$ with a peak centred on $\sim 8.1 \mathrm{~h}$ and a width of 0.41 in $\log \left(P_{\text {orb }}[d]\right)$ as determined from a Gaussian fit.

\section{Analysis}

We performed a radial velocity survey of 1246 WDMS binaries. The majority of the systems have three radial velocity measurements, spread over hours to hundreds of days (see Fig. 1). We have identified 191 of these systems as PCEBs based on the detection of significant radial velocity variations. While we consider the remaining 1055 systems to be candidate wide binaries, it is clear that there will still be a number of PCEBs among them that our radial velocity survey failed to identify because of observational selection effects.

Our ultimate aim is to establish the intrinsic period distribution of the SDSS PCEBs. We first investigate in the following sections what information might be gleaned from the distribution of probabilities of our initial radial velocity survey (Fig. 2). Subsequently, we analyse the observational biases within the orbital period distribution of the SDSS PCEBs (Fig. 7), and derive a bias-corrected period distribution, which will then be discussed in Sect. 6 in the context of compact binary evolution.

\subsection{Does our radial velocity survey constrain the orbital period distribution of WDMS binaries?}

The detailed shape of the probability distribution used to identify PCEBs among SDSS WDMS binaries will depend on the technical details of our radial velocity survey (spectral resolution, temporal sampling, number of spectra) and the intrinsic period distribution of the WDMS binaries. To test how sensitive the observed probability distribution is to differences in the underlying orbital period distribution, we performed extensive Monte Carlo simulations. As input, we used the measured stellar masses (Table 5), the times of observation, and the radial velocities and associated errors (Table 3 ) for all 1246 WDMS binaries within our radial velocity survey. We then adopted four different orbital period distributions: (1) short-period binaries only, with $2 \mathrm{~h}<P_{\text {orb }}<100 \mathrm{~d}$, and uniformly distributed in $\log \left(P_{\text {orb }}\right)$; (2) wide binaries only, with $3.5<\log \left(P_{\text {orb }}[\mathrm{d}]\right)<6.5$, uniformly distributed in $\log \left(P_{\text {orb }}\right)$; (3) a bi-modal distribution with a shortperiod binary population peaking at $7.5 \mathrm{~h}$, normally distributed in $\log \left(P_{\text {orb }}\right)$ and with a minimum period of $\sim 1.5 \mathrm{~h}$, plus a second population of wide binaries with $3.5<\log \left(P_{\text {orb }}[\mathrm{d}]\right)<7.5$, uniformly distributed in $\log \left(P_{\text {orb }}\right)$, and finally (4) a uniform period distribution in $\log \left(P_{\text {orb }}\right)$ with $\log \left(P_{\text {orb }}[\mathrm{d}]\right)<8.5$. Obviously (1), (2) and (4) are not very likely to be physically relevant, but were adopted to run the test with several radically different period distributions. We then used $10^{4}$ random inclinations per object, uniformly distributed in $\cos i$, and calculated the probability distributions of the two adopted period distributions. We assumed circular orbits for simplicity (and note that the orbits of PCEBs will be, in most cases, circularised).

The results of this simulation are illustrated in Fig. 8, showing the adopted period distributions on the left-hand side, and the resulting probability distributions on the right-hand side, with the observed probability distribution shown for comparison. The top two panels show that a peak at $P\left(\chi^{2}\right)=1$ requires close binaries, whereas a broad accumulation towards $P\left(\chi^{2}\right)=0$ is dominated by wide binaries. In other words, the observed probability distribution (Fig. 2) can only be explained if the underlying 


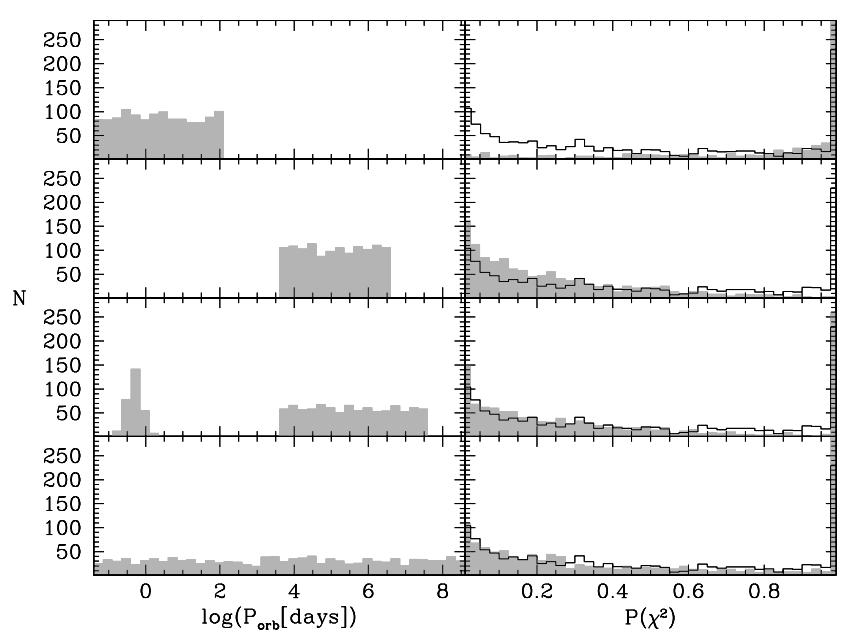

Fig. 8. Assumed orbital period distributions (left panels) and associated simulated distribution of the probability of measuring large radial velocity variations (grey) with measured distribution in black for comparison (right panels).

orbital period distribution of the observed WDMS binaries contains both wide binaries $\left(P_{\text {orb }} \gtrsim 10^{3} \mathrm{~d}\right)$ and PCEBs with shorter periods $\left(P_{\text {orb }} \lesssim 100 \mathrm{~d}\right)$.

However, the bottom two panels show that the two period distributions that contain both short-period PCEBs and wide binaries, but radically differ in shape, both lead to probability distributions that are very similar to the observed one. We conclude that our radial velocity survey carried out to identify PCEBs alone cannot constrain the detailed shape of the underlying WDMS binary orbital period distribution, and that it is therefore necessary to accurately measure the orbital periods for a large and homogeneous sample of PCEBs.

\subsection{Observational biases and selection effects}

We have presented in Sect. 4.2.4 the orbital period distribution of 79 PCEBs that were spectroscopically identified by SDSS (Fig. 7). The resulting orbital period distribution peaks at $\sim 8 \mathrm{~h}$ and very few systems with orbital periods longer than a day have been found.

This is not only the largest but also the most homogeneous sample of PCEBs available so far, and it has a strong potential to provide crucial constraints on the theories of close compact binary formation and evolution (e.g. Schreiber et al. 2010; Zorotovic et al. 2010; Rebassa-Mansergas et al. 2011b). It is, however, fundamental to understand any observational selection effects that may affect the properties of the SDSS PCEB sample. In the context of the orbital period distribution, there are two particular points that need to be investigated. Firstly, our radial velocity PCEB identification method is obviously more sensitive to short orbital period and high inclination systems, and secondly, we only measured the orbital periods of a subsample of the identified PCEBs, which is not necessarily representative of the entire sample.

\subsubsection{PCEB identification}

While we have compiled the largest and most homogeneous sample of PCEBs to date, not all the systems in this sample have been identified in the same way. Ten systems were initially

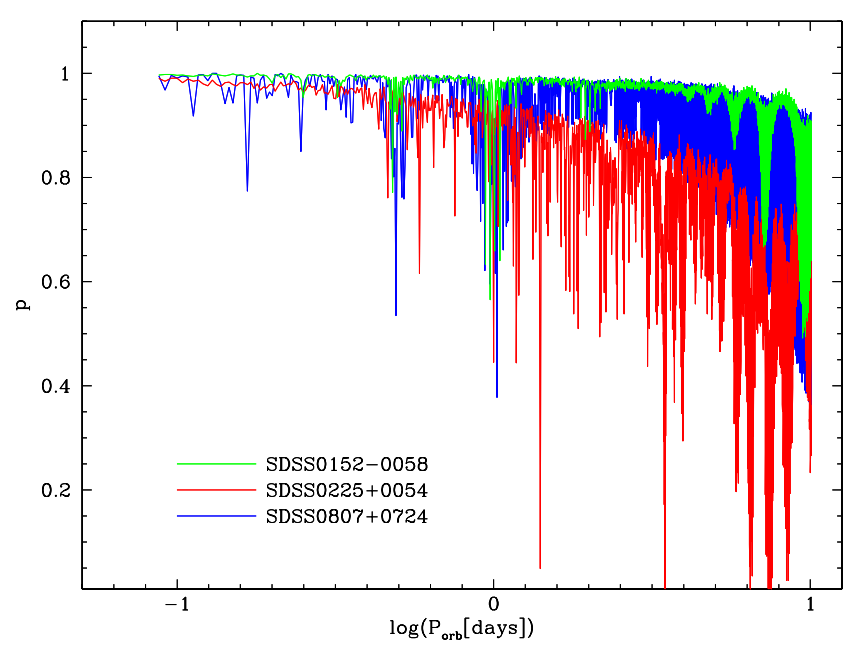

Fig. 9. Detection probability for three cases corresponding to 2 spectra taken at the VLT (green), 6 spectra taken at the WHT, Magellan and SDSS (blue), and 16 spectra from the SDSS (red).

classified as PCEBs because of photometric variability ${ }^{6}$ and four because of radial velocity variations seen over the course of a single night ${ }^{7}$. Both methods favour the identification of short orbital period PCEBs. This is illustrated in Fig. 7, where all those 14 systems have periods $\$ 9 \mathrm{~h}(0.4 \mathrm{~d})$. We discard them in the following analysis.

The remaining 65 PCEBs were identified by radial velocity variations from spectra that have been obtained on different nights, and we will concentrate our analysis of the observational biases on this "core" sample. The strength of the observational bias introduced in a radial velocity search for binaries depends on the intrinsic orbital period and the sampling frequency.

We performed Monte-Carlo simulations similar to those presented in Schreiber et al. (2008, 2010) and Rebassa-Mansergas et al. (2008) to evaluate if the observed orbital period distribution (Sect. 4.2.2) is significantly biased by the design of our radial velocity survey, and we calculated the detection probability as a function of orbital period for all 65 PCEBs. Given the times of observation and the error of the radial velocity measurements, we randomly select $10^{4}$ phases and inclinations for a given orbital period, and determine the corresponding $\chi^{2}$ probability. The PCEB detection probability for each system as a function of the orbital period is then simply given by the number of cases with $P\left(\chi^{2}\right)>0.9973(>3 \sigma)$ divided by $10^{4}$. The resulting detection probabilities depend on the number of spectra taken, the temporal sampling of the measurements, and the accuracy of the radial velocity measurement (i.e. basically the spectral resolution of the telescope/instrument combination that has been used).

Figure 9 shows the obtained detection probabilities for three rather extreme examples: SDSS 0807+0724 $\left(P_{\text {orb }}=\right.$ $11.45 \mathrm{~h}$, identified as a PCEB from two VLT/FORS spectra), SDSS 0152-0058 $\left(P_{\text {orb }}=2.15 \mathrm{~h}, 3 \times \mathrm{WHT}, 2 \times\right.$ Magellan, $1 \times$ SDSS $)$, and SDSS $0225+0055\left(P_{\text {orb }}=21.86 \mathrm{~h}, 16 \mathrm{SDSS}\right.$ subspectra). We have chosen these three cases to illustrate that our PCEB identification method is sensitive to PCEBs with orbital periods in the range of 1-10 days (detection probabilities

\footnotetext{
6 SDSS 0106-0014, 0110+1326, 0303+0054, 0908+0604, 1212$0123,1423+2409,1548+4057,1648+2811,1724+5620$, and $2112+$ 1014.
}

7 SDSS 0307+3848, 1300+1908, 1348+1834, and 1625+6400. 


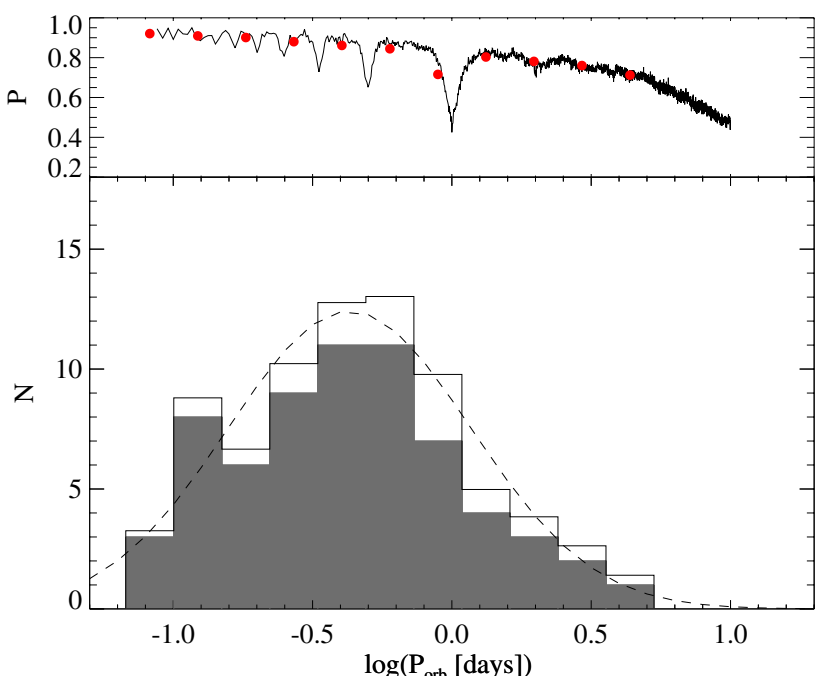

Fig. 10. Orbital period distribution of the 65 SDSS PCEBs discovered in an homogeneous way through radial velocity variations measured in spectra spread over different nights (grey) and the bias-corrected distribution (white). The Gaussian fit to the bias-corrected distribution is plotted with a dashed line. In the upper panel we show the mean probability of detection as a function of the orbital period (see text) and the corresponding mean value at each bin centre (red dots).

$\left.P\left(\chi^{2}\right) \gtrsim 0.35\right)$, as long as the time baseline exceeded one night. In addition, Fig. 9 illustrates the following: (1) the lower the resolution, the lower is the detection probability, even in the most favourable case, i.e. a large number of spectra; (2) variations in the detection probability with orbital period are dominated by phase coverage and are therefore strongest when only a few spectra are available. Orbital periods near one day and their first subharmonics $(12 \mathrm{~h}, 8 \mathrm{~h}$ ) have substantially depressed detection probabilities, as this increases the chances of sampling the same orbital phase twice on two subsequent nights; (3) superimposed on the fine structure related to the details of the sampling is a decreasing envelope, which is caused by a wider range of (low) inclinations leading to radial velocity variations that are too low to be resolved. The mean detection probability for the whole sample, calculated from the 65 individual detection probabilities, is higher than $35 \%$ up to ten day orbital periods (Fig. 10, upper panel), demonstrating that our radial velocity survey is capable of identifying PCEBs with long orbital periods. We use this mean detection probability to bias-correct the observed orbital period distribution in Sect. 5.2.3.

\subsubsection{PCEBs selected for orbital period measurements}

Given that we have measured orbital periods only for a fraction of the PCEBs that we identified, the next question to address is whether the subset of systems is representative of the whole sample. For instance, our follow-up strategy might have been biased towards the "easier" targets, i.e. those that exhibited large variations in our radial velocity survey. For this purpose, we compared the cumulative radial velocity variations of the 65 PCEBs identified in our radial velocity survey (see Sect. 4.1) and of those PCEBs for which we obtained no additional followup observations (Fig. 11). A Kolmogorov-Smirnov test between both sets gives a probability of 0.97 of both samples being drawn from the same parent population. We can conclude that both distributions are very similar and that we have measured the orbital period of a representative subsample of the SDSS PCEB population.

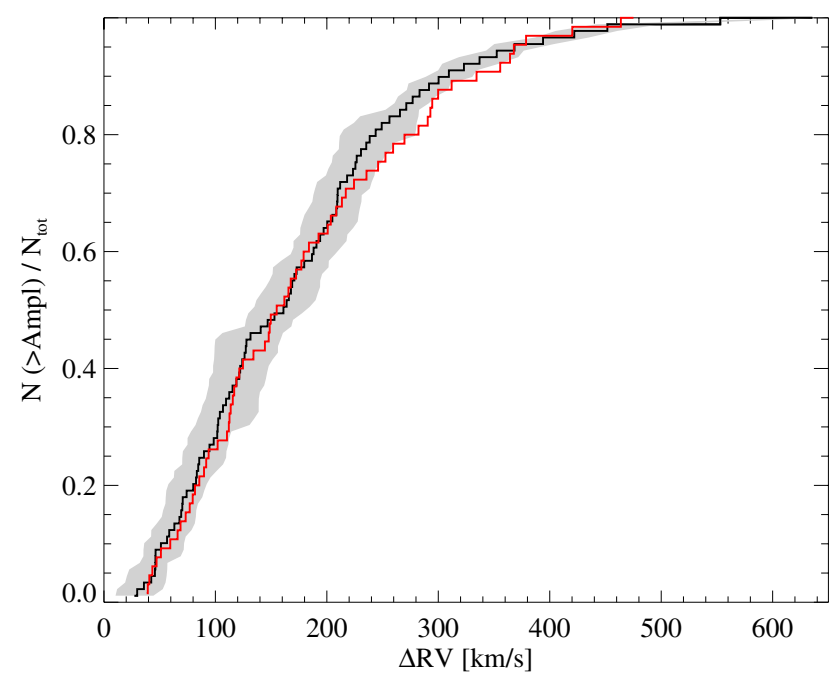

Fig. 11. Cumulative distribution function $(\mathrm{CDF})$ of the radial velocity variations used for the identification of PCEBs, systems with measured orbital period are shown in red and systems that were not further followed-up are shown in black. The grey area is the corresponding $\mathrm{CDF}$ taking the $1 \sigma$ radial velocity errors into account.

\subsubsection{Bias corrected orbital period distribution}

We have demonstrated in Sect.5.2.2 that the 65 PCEBs that were identified from multiple-night radial velocity snapshots and have measured orbital periods are representative of the entire SDSS PCEB sample. Here, we apply the average detection probability (Sect. 5.2.1) to correct their observed period distribution (Fig. 10). It is evident that the observational bias of our radial velocity survey does not dramatically affect the conclusions drawn in Sect. 4.2.4, i.e. the period distribution of the SDSS PCEB sample broadly follows a normal distribution centred at $\sim 10.3 \mathrm{~h}$ (0.43 d), a width of 0.44 in $\log \left(P_{\text {orb }}[d]\right)$, a short-period cut-off at $\sim 2 \mathrm{~h}$ and a long-period cut-off at $\sim 5 \mathrm{~d}$, where the position of the peak and the width have been determined from a Gaussian fit. The bias-corrected period distribution is discussed in the context of published binary population models in Sect. 6.4.

\section{Discussion}

\subsection{The PCEB fraction among WDMS binaries}

By analysing the radial velocity variations of 1246 SDSS WDMS binaries we identified 191 PCEBs (Sect. 4.1), which provides a lower limit of $\sim 15 \pm 1 \%$ to the fraction of PCEBs among the SDSS WDMS binaries. However, for a substantial number of these 1246 systems, the only available source of radial velocities are the SDSS subspectra, which, as they are usually taken during a single night (see Fig. 1), are only sensitive to very short orbital periods.

If we only consider the subsample of 718 WDMS binaries that were observed on different nights, we find 164 PCEBs, i.e. a PCEB fraction of $\sim 23 \pm 2 \%$. This number is similar to the one found by Schreiber et al. (2008), based on our VLT/FORS radial velocity survey of 26 SDSS WDMS binaries. This empirical determination of the PCEB fraction is broadly consistent with the results of the binary population synthesis carried out by Willems \& Kolb (2004).

Two observational selection effects will affect this empirical estimate of the PCEB fraction. On the one hand, our analysis in Sect. 5.2.1 showed that a number of PCEBs will escape 
A. Nebot Gómez-Morán et al.: The orbital period distribution of SDSS PCEBs. XII.

identification because of their low orbital inclination. From the mean detection probability and the bias-corrected orbital period distribution we learn that $\sim 16 \%$ of the PCEBs are not detected owing to a combination of inclination and phase coverage (see Fig. 10). Assuming that the 164 detected PCEBs (observed in different nights) follow the same orbital period distribution as the observed one (drawn from 65 of them) the number of expected PCEBs is 195, implying a fraction of PCEB to WDMS binaries of $27 \pm 2 \%(195 / 718)$. On the other hand, WDMS binaries that are sufficiently wide will appear as blended objects, or even fully resolved objects, and not have been followed up spectroscopically, because they have unreliable colours or colours of single white dwarfs and M-dwarfs, respectively. As a result, our estimate of $27 \pm 2 \%$ PCEBs among the spatially unresolved SDSS WDMS binaries can be taken as an upper limit on the intrinsic fraction of PCEBs among all WDMS. Schreiber et al. (2010) estimated the fraction of SDSS resolved WDMS to be $12-23 \%$. Taking this into account we finally estimate the intrinsic fraction of PCEBs to be $\sim 21-24 \%$.

Farihi et al. (2006, 2010) pursued a complementary approach to investigate the make-up of the known WDMS binary population, obtaining high spatial resolution imaging with HST/ACS. They observed a total of 72 WDMS binaries unresolved from the ground, and found a bimodal distribution in projected binary separations: all spatially resolved systems had binary separations $\gtrsim 10 \mathrm{AU}$, clearly above the detection threshold of 1-2 AU. After accounting for the frequency of WDMS binaries that can be resolved from the ground Farihi et al. $(2005,2010)$ conclude that the fraction of short-period binaries among all WDMS binaries is $\simeq 25 \%$, which is in excellent agreement with our estimate presented here. Nineteen of the WDMS binaries imaged with HST were also spectroscopically re-discovered by SDSS, and are thus in our WDMS binary sample (Table 7).

Farihi et al. (2010) predict that all the WDMS binaries unresolved by HST (Table 5 in their paper) should be short-period PCEBs, which is confirmed for all five systems for which we have obtained follow-up observations, spanning more than one night. For an additional two of Farihi's unresolved systems (SDSS1341+6026, 1500+1659), we only have the radial velocity information of the SDSS subspectra spanning less than one hour, and we classified both systems as wide binary candidates. This underlines what we said above, i.e. that the single-night SDSS subspectra allow us to identify only the shortest-period WDMS binaries (which we fully take into account by our Monte Carlo analysis of the selection biases). For completeness, we note that one additional unresolved binary from Farihi et al. (2010) has recently been found to be an eclipsing WDMS binary with an orbital period of $2.93 \mathrm{~h}$ Drake et al. (2010).

Vice versa, based on our radial velocity information for nine of the WDMS binaries spatially resolved by HST (Table 4 of Farihi et al. 2010), we classified all of them as wide binary candidates (labelled as "wide" in the bottom portion of Table 7).

\subsection{PCEBs and detached CVs in the orbital period gap}

The observed orbital period distribution of CVs presents a sharp decrease in systems between 2-3h (Warner 1976; Whyte \& Eggleton 1980; Knigge 2006), referred to as the orbital period gap of CVs, which is interpreted as a key to understanding their long-term evolution. The mass ratio of CVs implies that they are undergoing stable mass transfer, and it is angular momentum loss that drives them towards shorter orbital periods, keeping the companion stars in Roche-lobe contact. Above the period gap, the secondary stars have a radiative core, and are subject
Table 7. SDSS-WDMS binaries in common with Farihi et al. (2010).

\begin{tabular}{|c|c|c|c|c|}
\hline WD-Name & SDSS-Name & Type & \# spec & $\Delta t[\mathrm{~d}]$ \\
\hline & Not resolved by HST & & & \\
\hline WD0303-007 & SDSS J030607.18 - 003114.4 & pceb & 4 & 2991 \\
\hline & $091143.09+22274$ & & & 5 \\
\hline WD $1051+5$ & SDSS J105421.97 + 51225 & & & 1089 \\
\hline & SDSS J114312.57 + 000926.5 & & & 2551 \\
\hline & & & & 0.03 \\
\hline & & & 3 & 1157 \\
\hline & & & 2 & 0.01 \\
\hline WD15 & $\begin{array}{r}\text { SDSS J152425.21 + } \\
\text { Resolved by HS }\end{array}$ & pceb & 3 & 1007 \\
\hline & SDSS J03002 & & 2 & 10 \\
\hline & & & & 0.03 \\
\hline & & wide & & 0.03 \\
\hline & & & & 0 \\
\hline & & & & 0 \\
\hline & $\mathrm{SDs}$ & wid & 3 & 0.03 \\
\hline & & & 4 & 3197 \\
\hline & & & 4 & 0.05 \\
\hline & & & 3 & 0.02 \\
\hline & & & 5 & 1438 \\
\hline WD1833 + 644 & SDSS J183329.18 + 643151.7 & wide & 9 & 43 \\
\hline
\end{tabular}

Notes. "Type" is our classification based on the radial velocity information. We list the number of spectra used for the classification and the time span between them. Two of the systems unresolved by HST only have SDSS subspectra taken within $<1 \mathrm{~h} .{ }^{(1)} P_{\text {orb }}=9.274 \mathrm{~h}$. (2) $P_{\text {orb }}=14.15 \mathrm{~h}$.

to magnetic wind braking that acts as a strong agent of angular momentum loss. The corresponding mass loss rates are sufficient to drive the companion stars of these long-period CVs out of thermal equilibrium. At $\sim 3 \mathrm{~h}$, the companion stars become fully convective, and magnetic wind braking is thought to stop. From there on, the evolution towards shorter periods is driven by gravitational wave radiation, resulting in much lower mass loss rates. Consequently, the secondary stars have time to relax to their thermal equilibrium radii, shrink within their Roche lobes, and turn into detached WDMS binaries with no mass transfer. Gravitational wave radiation keeps driving these detached systems towards shorter periods. At $\sim 2 \mathrm{~h}$ the secondary stars reestablish Roche-lobe contact, mass transfer starts again, and the systems re-appear as CVs. This "disrupted magnetic braking" scenario is currently the standard paradigm of $\mathrm{CV}$ evolution (Rappaport et al. 1983).

Observationally, there is very little difference between genuine pre-CVs that will start mass transfer within, or shortly below, the CV period gap, and those detached CVs (dCVs) that are crossing the gap. The common hallmark of both populations is that they are WDMS binaries with secondary spectral types $\sim$ M6 to $\sim$ M4. A very simple, but so far untested, prediction of the disrupted magnetic braking scenario is that there should be an excess of systems with periods of $2-3 \mathrm{~h}$ in the period distribution of WDMS binaries, owing to dCVs that are crossing the orbital period gap. Davis et al. (2008) carried out a number of binary population studies to estimate the strength of this effect, and predict that within the $\mathrm{CV}$ period gap the number of dCVs should exceed that of genuine pre-CVs with secondary stars in the mass range $0.17-0.36 M_{\odot}$ by a factor $\sim 4-13$, which implies an increase in systems (PCEBs plus dCVs) of 1.5-2 in the gap in comparison with PCEBs at longer periods (see their Figs. 4 and 10). 


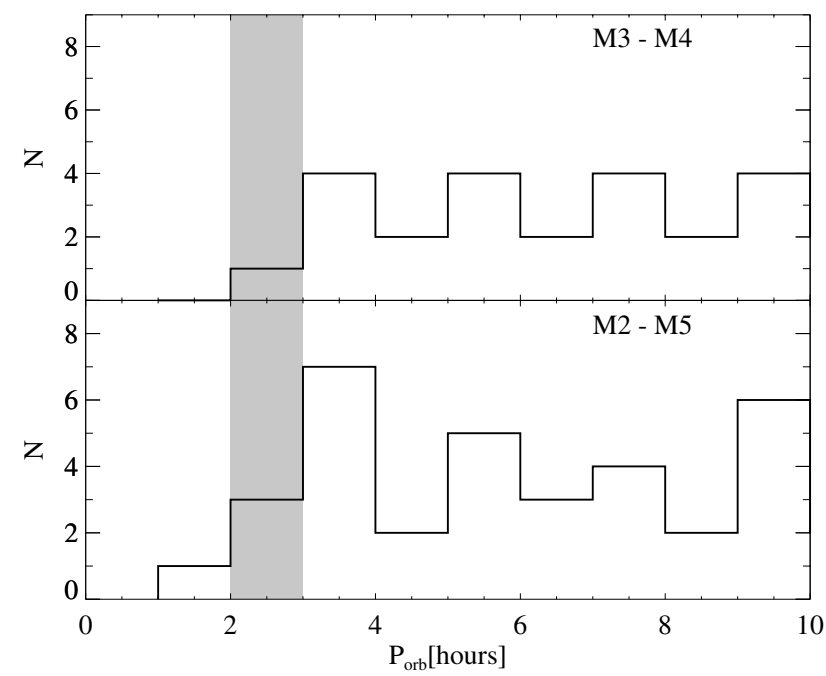

Fig. 12. Orbital period distribution of PCEBs with secondary spectral types in the range M3-M4 (upper panel) and M2-M5 (bottom panel). Magnetic braking disruption predicts a peak at around $2-3 \mathrm{~h}$ corresponding to an increase in systems due to detached CVs, we highlighted the region in grey.

The large sample of SDSS PCEBs provides a first opportunity to test this prediction. Observationally, the secondary stars in the SDSS WDMS binaries are characterised in terms of their spectral type. CVs just above and below the period gap have $\sim$ M3-4, and $\sim$ M5 secondary stars, respectively. We show in Fig. 12 the period distributions of all SDSS WDMS binaries with M3-4 companions (top panel), and a somewhat broader range of M2-5 (bottom panel), which accounts, somewhat conservatively, for the uncertainty in the spectral types of the companion stars. The expected increase towards shorter periods by a factor of 1.5-2 is not present in our data. In contrast, the number of dCVs plus PCEBs is slightly decreasing (M3-4) or constant (M2-5), although the number of systems in this subsample is still rather small. Comparing the number of dCVs plus PCEBs in the gap with the number of PCEBs between 3 and $4 \mathrm{~h}$, we obtain a fraction of dCVs plus PCEBs (in the gap) to PCEBs (above the gap) of $0.25 \pm 0.30$ (M3-4) or $0.43 \pm 0.30$ (M2-5), which excludes the predicted increase of $1.5-2$ on a $3 \sigma$ (M3-4) or a $2 \sigma(\mathrm{M} 2-5)$ level.

Three SDSS PCEBs that match the expected characteristics of dCVs are SDSS 0052-0053, SDSS 1611+4640, and SDSS $2243+3122$, with orbital periods $2.74,1.98$, and $2.87 \mathrm{~h}$, respectively. The Roche-lobe filling factors of SDSS 0052-0053 and SDSS $1611+4640$ are $1.0 \pm 0.6$ and $1.52 \pm 0.50$, respectively. For SDSS $2243+3122$ we have no estimate of the (DC) white dwarf mass, and thus cannot determine the filling factor.

\subsection{Comparison with non SDSS PCEBs}

In this section we compare the orbital period distribution of PCEBs discovered within the SDSS, with that of PCEBs that were discovered by other surveys. We compiled a list of 48 nonSDSS PCEBs containing a white dwarf plus a main sequence star from Ritter \& Kolb (2003, version v7.14) and Brown et al. (2011). The orbital period distribution of non-SDSS PCEBs (Fig. 13) is overall similar to that of the SDSS PCEBs, presenting both a peak at around $\sim 5 \mathrm{~h}$ and decreasing number of systems towards long orbital periods. A Kolmogorov-Smirnov test between the cumulative period distributions (bottom panel

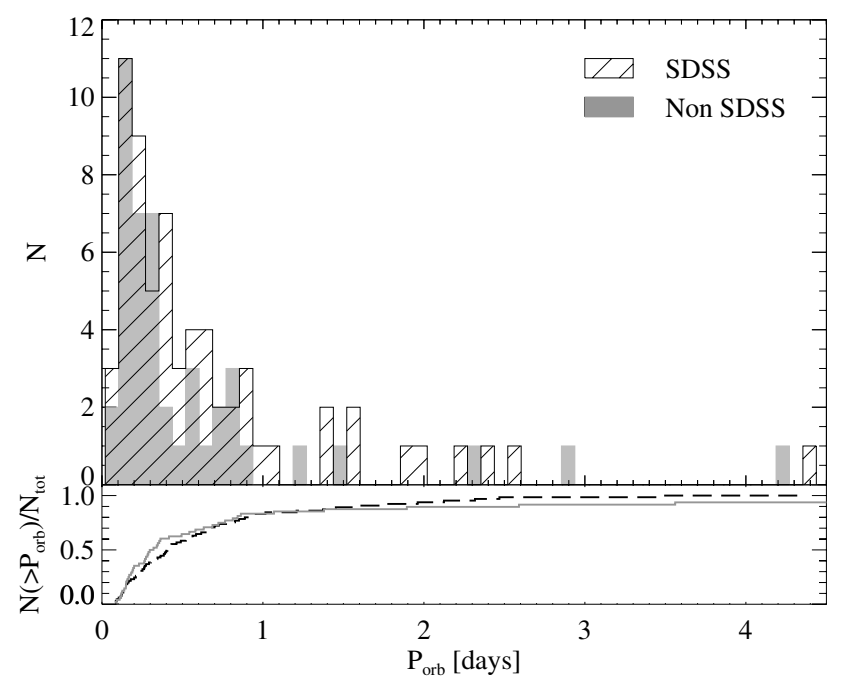

Fig. 13. Orbital period distribution of the 48 non-SDSS PCEBs that contain a white dwarf plus a main sequence star (grey histogram) and the 65 systems SDSS PCEBs discovered through radial velocity variations on spectra taken on different nights (dashed histogram) in days. Bottom panel: cumulative distribution function of non SDSS PCEBs (grey line) and SDSS PCEBs (black dashed line).

in Fig. 13) gives a probability of $35 \%$, i.e. consistent with the two samples being drawn from the same parent population.

We note that this result agrees with the conclusions of Miszalski et al. (2009), who first noticed the resemblance between the orbital period distribution of binary central stars in planetary nebulae (PNe) and the one of PCEBs as presented by Rebassa-Mansergas et al. (2008). This was later confirmed by Hillwig (2011), who selected 27 WDMS binaries that are the central stars of planetary nebulae (PNe) and the 35 SDSS WDMS binaries (as published by Zorotovic et al. 2010). Surveys dedicated to measuring the orbital period of the binary central stars of PNe have mostly been photometric (see Bond 2000, and references therein), implying a bias towards short orbital periods. De Marco et al. (2008) studied the possible biases introduced in the photometric sample, and conclude that the decrease in the number of PNe towards long periods is unlikely to come from observational biases.

\subsection{Comparison with predictions from BPS models}

In this section we discuss the SDSS WDMS binary sample in the context of published binary population synthesis models. However, it is necesary to keep in mind that the SDSS PCEB sample is subject to selection effects; i.e. systems with companion spectral types earlier than $\sim \mathrm{M} 0$ are severely underrepresented, and a comparison of the predicted orbital period distribution of the different BPS studies for the corresponding mass domain is in most cases not possible.

Common to all BPS models is the prediction that the population of WDMS binaries should be made up of wide binaries, with orbital periods longer than one year, in which the components never interacted, and close binaries that formed from commonenvelope evolution. The two most important parameters determining the outcome of the $\mathrm{CE}$ phase are the initial mass ratio distribution of the progenitor binaries and the efficiency of the ejection of the common-envelope, $\alpha_{\mathrm{CE}}$, which will affect the position of the peak in the orbital period distribution of the PCEBs and the slope towards longer orbital periods. 
One of the first BPS for WDMS binaries was carried out by de Kool (1992) and de Kool \& Ritter (1993), predicting that most PCEBs should have periods shorter than about one day. An extended tail towards longer orbital periods up to 1000 days is expected for $\alpha_{\mathrm{CE}}=1$, while no tail is observed for $\alpha_{\mathrm{CE}}=0.3$. The authors also simulated the expected properties of a volume limited blue sample of present-day WDMS binaries; however, comparison with the very small number of PCEBs at that time did not lead to any significant observational constraints. Our orbital period distribution (Fig. 10) is very similar to their predicted one for $\alpha_{\mathrm{CE}}=0.3$, and the mass of the secondary star picked randomly from the IMF, already pointed out by Miszalski et al. (2009) in the context of PN, or with $\mathrm{d} N \propto \mathrm{d} q$ (see their Fig. 12b). However, this comparison should be regarded with some caution, as de Kool \& Ritter (1993) simulated their "observational" sample for the selection criteria of the Palomar-Green Survey (Green et al. 1986) $(U<16$ and $U-B<-0.5)$, whereas the SDSS WDMS sample has a much larger limiting magnitude $i \simeq 19.1$ and is drawn from a much larger ugriz colour volume.

Willems \& Kolb (2004) carried out an extensive BPS study of WDMS binaries, using $\alpha_{\mathrm{CE}}=1$, for different initial mass ratio distributions. Their BPS predicts that the orbital period distribution of PCEBs peaks around one day, with a substantial tail towards longer orbital periods of up to 100 days, which contrasts to the period distribution of the SDSS PCEBs (Fig. 10). However, Willems \& Kolb (2004) did not convolve their model WDMS binary population with detailed observational selection effects, but just discussed their results in terms of luminosity ratio distributions, $L_{\mathrm{wd}} / L_{\mathrm{MS}}$, which prevents any more quantitative comparison with our SDSS WDMS binary sample.

Politano \& Weiler (2007) investigated the effects on the population of PCEBs for $\alpha_{\mathrm{CE}}$ depending on the mass of the secondary star. They predict an orbital period distribution peaking at around three days, which is incompatible with the observed period distribution of the SDSS WDMS binaries. However, Politano \& Weiler (2007) did not simulate a realistic "observational" WDMS population, again preventing a quantitative comparison with our sample.

The most recent BPS of WDMS binaries has been performed by Davis et al. (2010), who, in contrast with previous works, calculate the binding energy parameter $\lambda$ from stellar evolution models incorporating the internal energy of the envelope. While this is a significant step forward compared to previous PBS that worked with a constant value of $\lambda$, Davis et al. (2010) obtained the values of $\lambda$ from the tables of Dewi \& Tauris (2000), which cover only a very small part of the relevant parameter space. The orbital period distribution of Davis et al. (2010) predicts a long tail of systems with periods $>1 \mathrm{~d}$, inconsistent with the period distribution of the PCEBs known at the time of publication (their Fig. 10), even when taking the detection probabilities of Rebassa-Mansergas et al. (2008) into account. Our more detailed observed (Fig. 7) and bias-corrected (Fig. 10) orbital period distributions are in better agreement with their predicted orbital period distribution limited to PCEBs with companion masses $\leq 0.5 M_{\odot}$, i.e. spectral types later than $\sim \mathrm{M} 0$ (see their Fig. 10 middle panel).

In summary, most published BPS models predict a substantial number of PCEBs with orbital periods $>1 \mathrm{~d}$, which is currently incompatible with the orbital period distribution of the SDSS PCEBs. The observed dearth of long-period PCEBs could be reconciled with a low value of $\alpha_{\mathrm{CE}}$. Zorotovic et al. (2010) used a subset of 35 of the PCEBs presented here, along with some other 25 non-SDSS PCEBs, to constrain the value of $\alpha_{\mathrm{CE}}$ by reconstructing their evolution. They found that there seems to be no dependence of $\alpha_{\mathrm{CE}}$ on the mass of the secondary star and that, if the value is universal, only a low value of $\alpha_{\mathrm{CE}}$ can explain the short orbital periods observed among the known PCEBs. However, it is also clear that even the SDSS PCEB sample is subject to selection effects, i.e. systems with companion spectral types earlier than $\sim \mathrm{M} 0$, i.e. masses $\gtrsim 0.5 M_{\odot}$ are severely underrepresented, which are expected to have longer orbital periods than the currently dominant population of PCEBs with low-mass (mid M-type) companions.

\section{Conclusions}

Nearly 2000 WDMS binaries have been spectroscopically identified within the SDSS survey. A detailed characterisation and analysis of this large and homogeneously selected sample promises to improve our understanding of close binary evolution substantially, in particular of the common-envelope phase. Using follow-up observations of 385 WDMS binaries, combined with the SDSS subspectra for an additional 861 systems, we identified 191 PCEBs. We determined orbital periods for 58 of these systems, increasing the total number of SDSS PCEBs with known orbital periods to 79 . We analysed the observational biases inherent to the SDSS PCEB sample, and found that $21-24 \%$ of all SDSS WDMS binaries with $\mathrm{M}$ dwarf companions must have undergone common-envelope evolution. The bias-corrected orbital period distribution of the SDSS PCEBs shows a peak near $\sim 10.3 \mathrm{~h}$, and drops fairly steeply to both shorter and longer periods, with no systems at $P_{\text {orb }}<1.9 \mathrm{~h}$, and few systems at $P_{\text {orb }}>1 \mathrm{~d}$. Comparing the properties of the SDSS PCEB population to the results of published binary population, we concluded that the observed dearth of long-period systems can be explained by low values of $\alpha_{\mathrm{CE}}$. Observational selection effects against earlier-type companions can also play a role. Given a Roche lobe-filling giant or asymptotic giant (the progenitor of the white dwarf component) in a binary system, the more massive the companion star, the greater the orbital energy available to eject the common-envelope, and therefore the longer the orbital period of the remnant PCEB, other things being equal. Unlocking the diagnostic potential of the SDSS WDMS binary population now requires the computation of a state-of-the-art binary population model that includes a realistic simulation of the observational selection effects inherent to this sample.

Acknowledgements. Based on observations collected at: the European Organisation for Astronomical Research in the Southern Hemisphere, Chile (078.D-0719，079.D-0531，080.D-0407， 082.D-0507，083.D-0862，085.D0974); the Gemini Observatory, which is operated by the Association of Universities for Research in Astronomy, Inc., under a cooperative agreement with the NSF on behalf of the Gemini partnership: the National Science Foundation (United States), the Science and Technology Facilities Council (United Kingdom), the National Research Council (Canada), CONICYT (Chile), the Australian Research Council (Australia), Ministério da Ciência e Tecnologia (Brazil) and Ministerio de Ciencia, Tecnología e Innovación Productiva (Argentina) (GS-2007B-Q-27, GS-2008A-Q-31, GS-2008B-Q-40, GS-2009A-Q-56); the Magellan Telescopes Baade and Clay, and the Irénée du Pont Telescope located at Las Campanas Observatory, Chile; the William Herschel Telescope, operated on the island of La Palma by the Isaac Newton Group in the Spanish Observatorio del Roque de los Muchachos of the Instituto de Astrofísica de Canarias; the IAC80 operated on the island of Tenerife by the Instituto de Astrofísica de Canarias in the Spanish Observatorio del Teide; and at the Centro Astronómico Hispano Alemán (CAHA) at Calar Alto, operated jointly by the Max-Planck Institut für Astronomie and the Instituto de Astrofísica de Andalucía.

Funding for the SDSS and SDSS-II has been provided by the Alfred P. Sloan Foundation, the Participating Institutions, the National Science Foundation, the US Department of Energy, the National Aeronautics and Space Administration, the Japanese Monbukagakusho, the Max Planck Society, and the Higher Education Funding Council for England. The SDSS Web Site is http://www . sdss.org/. 
The authors would like to thank the referee, R. Webbink, for carefully reading the manuscript and for providing useful remarks that helped improve the quality of the paper. We would like to thank T. Forveille and B. Miszalski for useful comments. A.N.G.M. acknowledges support by the Centre National d'Études Spatial (CNES, ref. 60015). B.T.G. was supported by a rolling grant from STFC. A.N.G.M. and M.R.S. acknowledge support by the Deutsches Zentrum für Luft und Raumfahrt (DLR) GmbH under contract No. FKZ 50 OR 0404. M.R.S. was also supported by FONDECYT (grant 1100782), and the Center of Astrophysics at the Universidad de Valparaiso. A.R.M. acknowledges financial support from FONDECYT (grant 3110049). This work was supported by the Deutsche Forschungsgemeinschaft (DFG) under contract numbers Schw 536/261, 29-1, 30-1, 31-1, 32-1, 33-1, and 34-1. J.S. acknowledges financial support from STFC in the form of an Advanced Fellowship.

\section{References}

Abazajian, K. N., Adelman-McCarthy, J. K., Agüeros, M. A., et al. 2009, ApJS, 182,543

Adelman-McCarthy, J. K., Agüeros, M. A., Allam, S. S., et al. 2008, ApJS, 175, 297

Agüeros, M. A., Anderson, S. F., Covey, K. R., et al. 2009, ApJS, 181, 444

Bertin, E., \& Arnouts, S. 1996, A\&AS, 117, 393

Bilíková, J., Chu, Y., Gruendl, R. A., \& Maddox, L. A. 2010, AJ, 140, 1433

Bond, H. E. 2000, in Asymmetrical Planetary Nebulae II: From Origins to Microstructures, ed. J. H. Kastner, N. Soker, \& S. Rappaport, ASP Conf. Ser., 199,115

Briggs, K. R., Napiwotzki, R., Maxted, P. F. L., \& Wheatley, P. J. 2007, in 15th European Workshop on White Dwarfs, ed. R. Napiwotzki, \& M. R. Burleigh, ASP Conf. Ser., 372, 497

Brown, J. M., Kilic, M., Brown, W. R., \& Kenyon, S. J. 2011, ApJ, 730, 67 Casali, M., Adamson, A., Alves de Oliveira, C., et al. 2007, A\&A, 467, 777 Chu, Y., Guerrero, M. A., Gruendl, R. A., \& Webbink, R. F. 2004, AJ, 127, 477 Davis, P. J., Kolb, U., Willems, B., \& Gänsicke, B. T. 2008, MNRAS, 389, 1563 Davis, P. J., Kolb, U., \& Willems, B. 2010, MNRAS, 403, 179 de Kool, M. 1992, A\&A, 261, 188

de Kool, M., \& Ritter, H. 1993, A\&A, 267, 397

De Marco, O., Hillwig, T. C., \& Smith, A. J. 2008, AJ, 136, 323

Delfosse, X., Forveille, T., Ségransan, D., et al. 2000, A\&A, 364, 217

Dewi, J. D. M., \& Tauris, T. M. 2000, A\&A, 360, 1043

Drake, A. J., Djorgovski, S. G., Mahabal, A., et al. 2009, ApJ, 696, 870

Drake, A. J., Beshore, E., Catelan, M., et al. 2010, ApJ, submitted [arXiv: 1009.3048$]$

Eggleton, P. P. 1983, ApJ, 268, 368

Farihi, J., Becklin, E. E., \& Zuckerman, B. 2005, ApJS, 161, 394

Farihi, J., Hoard, D. W., \& Wachter, S. 2006, ApJ, 646, 480

Farihi, J., Hoard, D. W., \& Wachter, S. 2010, ApJS, 190, 275

Gänsicke, B. T., Araujo-Betancor, S., Hagen, H.-J., et al. 2004, A\&A, 418, 265

Green, R. F., Schmidt, M., \& Liebert, J. 1986, ApJS, 61, 305

Hambly, N. C., Collins, R. S., Cross, N. J. G., et al. 2008, MNRAS, 384, 637

Heller, R., Homeier, D., Dreizler, S., \& Østensen, R. 2009, J. Phys. Conf. Ser., 172,012023

Hewett, P. C., Warren, S. J., Leggett, S. K., \& Hodgkin, S. T. 2006, MNRAS, 367,454

Hillwig, T. C. 2011, in Asymmetric Planetary Nebulae 5 Conference

Hjellming, M. S., \& Taam, R. E. 1991, ApJ, 370, 709

Hodgkin, S. T., Irwin, M. J., Hewett, P. C., \& Warren, S. J. 2009, MNRAS, 394, 675

Horne, K. 1986, PASP, 98, 609

Kleinman, S. J., Harris, H. C., Eisenstein, D. J., et al. 2004, ApJ, 607, 426

Knigge, C. 2006, MNRAS, 373, 484

Koester, D., Napiwotzki, R., Voss, B., Homeier, D., \& Reimers, D. 2005, A\&A, 439, 317

Koester, D., Kepler, S. O., Kleinman, S. J., \& Nitta, A. 2009, J. Phys. Conf. Ser., 172,012006

Lawrence, A., Warren, S. J., Almaini, O., et al. 2007, MNRAS, 379, 1599

Liu, Q., \& Yang, Y. 2003, Chin. J. Astron. Astrophys., 3, 142

Maccacaro, T., Gioia, I. M., Wolter, A., Zamorani, G., \& Stocke, J. T. 1988, ApJ, 326,680

Marsh, T. R. 1989, PASP, 101, 1032

Martin, D. C., Fanson, J., Schiminovich, D., et al. 2005, ApJ, 619, L1
Maxted, P. F. L., Marsh, T. R., Moran, C. K. J., \& Han, Z. 2000, MNRAS, 314, 334

Michel, L., Herent, O., Motch, C., Pye, J., \& Watson, M. G. 2004, in Astronomical Data Analysis Software and Systems (ADASS) XIII, ed. F. Ochsenbein, M. G. Allen, \& D. Egret, ASP Conf. Ser., 314, 570

Miszalski, B., Acker, A., Moffat, A. F. J., Parker, Q. A., \& Udalski, A. 2009, A\&A, 496, 813

Motch, C., Guillout, P., Haberl, F., et al. 1998, A\&AS, 132, 341

Nebot Gómez-Morán, A., Schwope, A. D., Schreiber, M. R., et al. 2009, A\&A, 495,561

Nelemans, G., \& Tout, C. A. 2005, MNRAS, 356, 753

Nelemans, G., Verbunt, F., Yungelson, L. R., \& Portegies Zwart, S. F. 2000, A\&A, 360, 1011

O’Dwyer, I. J., Chu, Y., Gruendl, R. A., Guerrero, M. A., \& Webbink, R. F. 2003, AJ, 125, 2239

Paczyński, B. 1976, in Structure and Evolution of Close Binary Systems, IAU Symp., 73, 75

Politano, M., \& Weiler, K. P. 2007, ApJ, 665, 663

Pourbaix, D., Knapp, G. R., Szkody, P., et al. 2005, A\&A, 444, 643

Pyrzas, S., Gänsicke, B. T., Marsh, T. R., et al. 2009, MNRAS, 394, 978

Pyrzas, S., Gaensicke, B. T., Brady, S., et al. 2011, MNRAS, accepted [arXiv: 1109.1171]

Rappaport, S., Joss, P. C., \& Verbunt, F. 1983, ApJ, 275, 713

Rebassa-Mansergas, A., Gänsicke, B. T., Rodríguez-Gil, P., Schreiber, M. R., \& Koester, D. 2007, MNRAS, 382, 1377

Rebassa-Mansergas, A., Gänsicke, B. T., Schreiber, M. R., et al. 2008, MNRAS, 390, 1635

Rebassa-Mansergas, A., Gänsicke, B. T., Schreiber, M. R., Koester, D., \& Rodríguez-Gil, P. 2010, MNRAS, 402, 620

Rebassa-Mansergas, A., Nebot Gomez-Moran, A., Schreiber, M. R., et al. 2011a, MNRAS, accepted [arXiv: 1110.1000 ]

Rebassa-Mansergas, A., Nebot Gómez-Morán, A., Schreiber, M. R., Girven, J., \& Gänsicke, B. T. 2011b, MNRAS, 413, 1121

Ricker, P. M., \& Taam, R. E. 2008, ApJ, 672, L41

Ritter, H., \& Kolb, U. 2003, A\&A, 404, 301

Sanduleak, N., \& Pesch, P. 1989, ApJS, 70, 173

Schreiber, M. R., \& Gänsicke, B. T. 2003, A\&A, 406, 304

Schreiber, M. R., Nebot Gomez-Moran, A., \& Schwope, A. D. 2007, in ASP Conf. Ser. 372, ed. A. Napiwotzki, \& M. R. Burleigh, 459

Schreiber, M. R., Gänsicke, B. T., Southworth, J., Schwope, A. D., \& Koester, D. 2008, A\&A, 484, 441

Schreiber, M. R., Gänsicke, B. T., Rebassa-Mansergas, A., et al. 2010, A\&A, 513, L7

Schwarzenberg-Czerny, A. 1996, ApJ, 460, L107

Schwope, A. D., Nebot Gomez-Moran, A., Schreiber, M. R., \& Gänsicke, B. T. 2009, A\&A, 500, 867

Silvestri, N. M., Hawley, S. L., West, A. A., et al. 2006, AJ, 131, 1674

Silvestri, N. M., Lemagie, M. P., Hawley, S. L., et al. 2007, AJ, 134, 741

Smolčić, V., Ivezić, Ž., Knapp, G. R., et al. 2004, ApJ, 615, L141

Southworth, J., Gänsicke, B. T., \& Schreiber, M. R. 2007, in 15th European Workshop on White Dwarfs, ed. R. Napiwotzki, \& M. R. Burleigh, ASP Conf. Ser., 372, 453

Steinfadt, J. D. R., Bildsten, L., \& Howell, S. B. 2008, ApJ, 677, L113

Stoughton, C., Lupton, R. H., Bernardi, M., et al. 2002, AJ, 123, 485

Szkody, P., Henden, A., Mannikko, L., et al. 2007, AJ, 134, 185

Tappert, C., Gänsicke, B. T., Schmidtobreick, L., Mennickent, R. E., \& Navarrete, F. P. 2007, A\&A, 475, 575

Townsley, D. M., \& Gänsicke, B. T. 2009, ApJ, 693, 1007

Tremblay, P., Bergeron, P., Kalirai, J. S., \& Gianninas, A. 2010, ApJ, 712, 1345

Voges, W., Aschenbach, B., Boller, T., et al. 1999, A\&A, 349, 389

Warner, B. 1976, in Structure and Evolution of Close Binary Systems, ed. P. Eggleton, S. Mitton, \& J. Whelan, IAU Symp., 73, 85

Webbink, R. F. 1984, ApJ, 277, 355

Webbink, R. F. 2008, in Astrophys. Space Sci. Lib. 352, ed. E. F. Milone, D. A. Leahy, \& D. W. Hobill, 233

Whyte, C. A., \& Eggleton, P. P. 1980, MNRAS, 190, 801

Willems, B., \& Kolb, U. 2004, A\&A, 419, 1057

Yanny, B., Rockosi, C., Newberg, H. J., et al. 2009, AJ, 137, 4377

York, D. G., Adelman, J., Anderson, J. E., et al. 2000, AJ, 120, 1579

Zorotovic, M., Schreiber, M. R., Gänsicke, B. T., \& Nebot Gómez-Morán, A. 2010, A\&A, 520, A86

Pages 13 to 20 are available in the electronic edition of the journal at http://www . aanda. org 

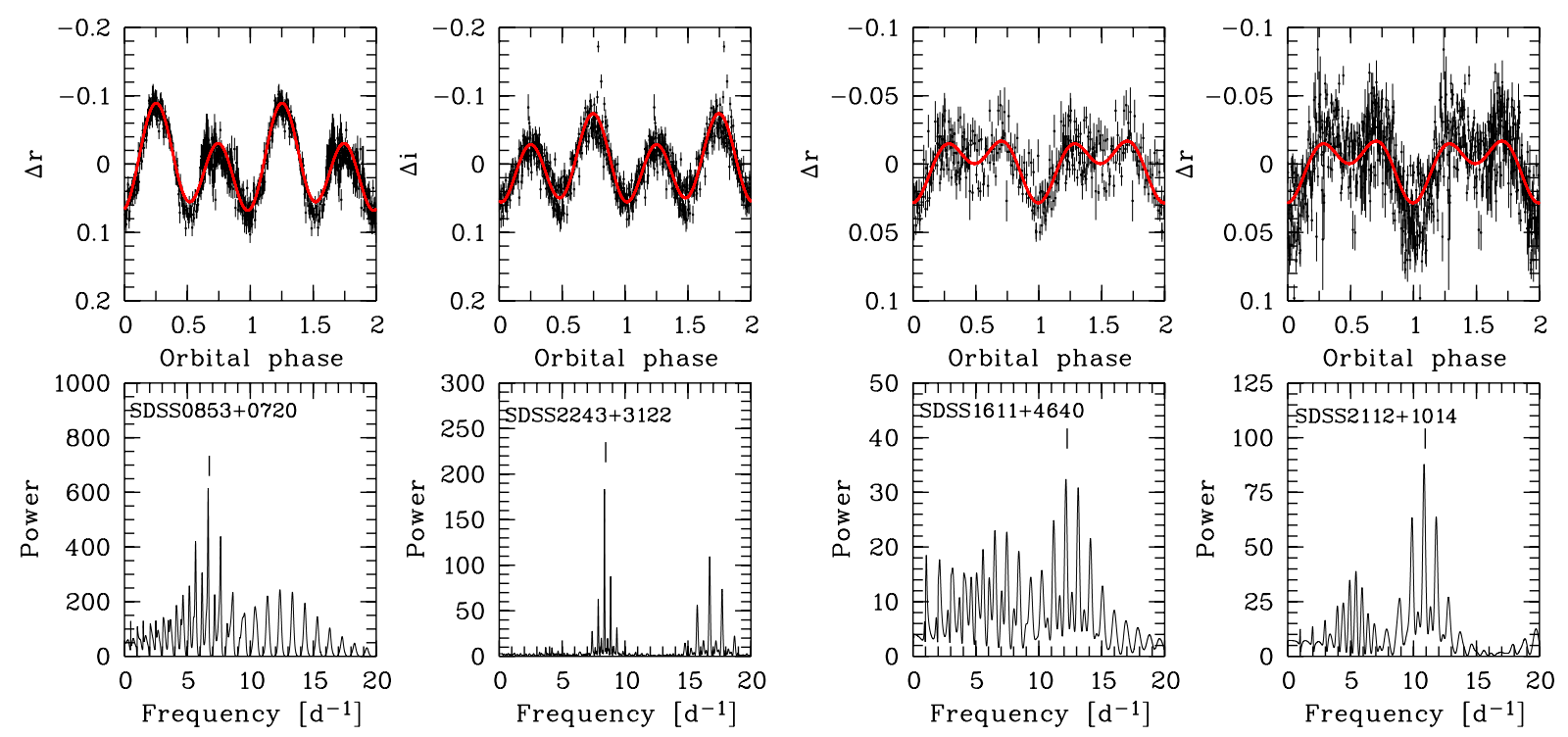

Fig. A.1. Photometric periodogram, and photometric light curves for SDSS0853+0720, SDSS 2243+3122, SDSS $1611+4640$ and SDSS $2112+1014$ phase folded with the orbital period corresponding to the highest peak in the periodogram (bottom panels), $P_{\text {orb }}=0.1503 \mathrm{~d}$, $P_{\text {orb }}=0.11954 \mathrm{~d}, P_{\text {orb }}=0.0823 \mathrm{~d}$ and $P_{\text {orb }}=0.0923 \mathrm{~d}$ respectively. Double-sine fits are shown in red.

\section{Appendix A: Optical light curves}

Photometric follow-up observations were carried out for a total of seven WDMS binaries where the available spectroscopy suggested a short orbital period. Telescope, filter, and duration of the observations are listed in Table 1. Four out of the seven observed systems showed ellipsoidal modulation: SDSS 0853+0720, SDSS 1611+4640, SDSS 2112+1014, and SDSS $2243+3122$. We determined their orbital periods by computing periodograms and fitting sine curves to the phase folded light curves (see Fig. A.1). We estimated $P_{\text {orb }}=3.6,1.9,2.2$, and $2.8 \mathrm{~h}$ respectively. Spectroscopic follow-up observations were carried out for SDSS 0853+0720, SDSS 1611+4640, and SDSS $2243+3122$ covering a full cycle, and confirming the measured periods. We could connect the spectroscopic and photometric runs for SDSS 1611+4640 and SDSS 2243+3122, without cycle count aliases, which allowed us to refine their orbital periods.

The light curves of SDSS $0853+0720$ and SDSS 2243+3122 both show two uneven maxima at phases 0.25 and 0.75 . For SDSS $0853+0720$ the first maximum is brighter than the second one, while for SDSS $2243+3122$ is the other way round. In both systems equal minima at phases 0 and 0.5 are observed, and the maximum variation is almost 0.2 mag in the $r$ and the $i$ bands, respectively. Ellipsoidal modulation and spots in one of the hemispheres of the secondary star could explain the uneven maxima (O'Connell effect, see Liu \& Yang 2003). Photometry carried out on 15 August 2009 for SDSS 2243+3122 revealed a flare with $\sim 25$ min length in the decay, and rising by $0.35 \mathrm{mag}$ in the $i$ band (see Fig. A.2). This would strengthen the argument of magnetic activity being the cause of the observed disparity in the maxima (see Fig. A.1).

Although no radial velocity curve is available for SDSS $2112+1014$, the five available spectra show radial velocity variations of more than $300 \mathrm{~km} \mathrm{~s}^{-1}$ in less than $40 \mathrm{~min}$, indicating a rather short orbital period, consistent with the measured photometric orbital period of $2.2 \mathrm{~h}$.

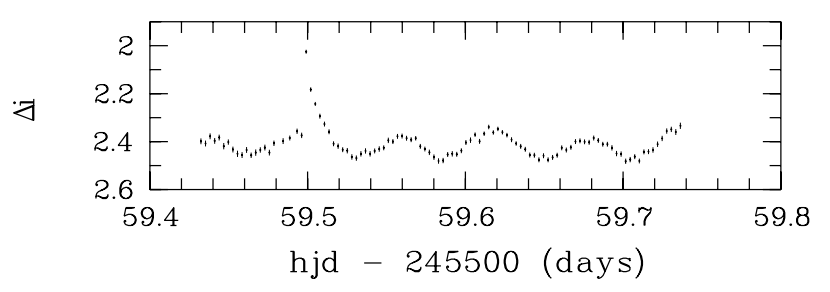

Fig. A.2. Differential photometry in the $i$ band obtained for SDSS $2243+2122$ on 15 August 2009 . A flare of $\sim 25$ min length with a relative enhancement of $\sim 0.4$ mag was observed.

\section{Appendix B: Notes on individual systems}

SDSS $0225+0054$, the primary star was classified as a DC by Kleinman et al. (2004). Although it is true that no absorption lines are visible in the primary star we consider that the signal-to-noise of the residual white dwarf after subtracting of the best-fitted secondary template is insufficient for any other classification than white dwarf (see Rebassa-Mansergas et al. 2007, for details on the spectral analysis). SDSS $0225+0054$ and SDSS 0853+0720 are WDMS binaries consisting of cold DC white dwarfs as primary stars and an M4.5 and M3 companion star, repectively. These are clear examples of the old PCEBs predicted by Schreiber \& Gänsicke (2003) that would not have been found in any search for blue objects.

SDSS 0307+3848 (SDSS J030716.44+384822.8) has a red star nearby - within $5^{\prime \prime}$ - (SDSS J030716.16+384821.4) and is slightly brighter in the $r$ band (16.70 versus 15.17$)$. To be sure that there was no contamination in our spectra by this source we rotated the slit so as to take spectra simultaneously and compared the radial velocities with those of this system. No variations in the radial velocity of this visual companion were observed. The optical spectrum of SDSS $0307+3848$ is dominated by the secondary star, and it presents strong emission lines. We measured the radial velocities from the $\mathrm{H} \alpha$ line in the SDSS-subspectra. 
The $\mathrm{Na} \mathrm{I}$ and the $\mathrm{H} \alpha$ lines are in phase, and the amplitudes were consistent with each other within the errors.

SDSS 1006+0044, SDSS 1523+4604, and SDSS 1844+4120 have very cold WDs, $T_{\text {eff }}=7800,8400,7600 \mathrm{~K}$, late secondary star spectral types M9, M7, M6, and are among the closest PCEBs in the sample, $d_{\mathrm{wd}}=60,76,73$ pc respectively. SDSS $1844+4120$ is the system with the highest systemic velocity, and it has Ca II H\&K in absorption; i.e., the white dwarf is a DAZ. This photospheric absorption could be produced by some wind from the secondary star that is being accreted by the WD (see e.g. LTT560 Tappert et al. 2007). Looking at the SDSS images of SDSS 1523+4604, aka CSO749 (Sanduleak \& Pesch 1989), the components of this binary seem to be slightly separated, also noted by Heller et al. (2009), and there seems to be a third red object towards the north-east. We measured an orbital period of $9.93 \mathrm{~h}$, so it could be that SDSS $1523+4604$ is a triple system formed by a close binary and a wide companion.

SDSS 1143+0009, aka WD1140+004, is an X-ray emitting source (Agüeros et al. 2009) from the Rosat All Sky Survey (Voges et al. 1999). The X-ray to optical flux ratio $\left(\log f_{\mathrm{X}} / f_{\mathrm{g}}\right)$ is 0.22 , a value too high to be explained by the coronal emission of the dMe star (see Maccacaro et al. 1988; Motch et al. 1998, for typical values), and the SDSS spectrum presents some marginal $\mathrm{H} \alpha$ emission (although this can be partially masked by the flux of the white dwarf). The high flux ratio could be explained by some wind from the secondary star being accreted by the white dwarf. If so, some Ca II H\&K absorption would then be expected, which is not visible in the spectrum. A small fraction of isolated white dwarfs show hard (higher than $0.5 \mathrm{keV}$ ) X-ray emission (O’Dwyer et al. 2003; Chu et al. 2004; Bilíková et al. 2010); nevertheless, the effective temperature of the white dwarf in SDSS $1143+0009(\sim 17000 \mathrm{~K})$ is too cold to explain its hard X-ray emission. Briggs et al. (2007) list six other PCEBs X-ray emitters: BPM 71214, RR Cae, UZ Sex, EG UMa, Feige 24, and WD 1541-381. We investigated the X-ray fluxes of these sources within the XMM-Newton, making use of $\mathrm{xcat}-\mathrm{DB}^{8}$ (Michel et al. 2004). None of them show an X-ray spectrum as hard as that of SDSS 1143+0009. It has a counterpart the Galaxy Evolution Explorer (GALEX) database (Martin et al. 2005), with detection only in the FUV flux, $17.97 \pm 0.08$ mag, consistent with the effective temperature of the white dwarf. We conclude that there is no straightforward explanation of the hard X-ray emission of SDSS $1143+0009$

SDSS $1352+0910$ contains a hot WD, $T_{\text {eff }}=36200 \mathrm{~K}$ and no companion visible in the SDSS spectrum. Nevertheless, emission in the $\mathrm{H} \alpha$ line was observed in the SDSS spectrum, pointing to a binary nature for the system, and triggering further spectroscopy, which revealed it to be a close binary. We investigated infrared surveys to learn more about the secondary star. We found a counterpart in the UKIDSS catalogue, ULAS J135228.14+091039.0. For details on the UKIDSS project, camera, photometric system, calibration, pipeline processing, and science archive see Lawrence et al. (2007); Casali et al. (2007); Hewett et al. (2006); Hodgkin et al. (2009) and Hambly et al. (2008). Magnitudes found in the seventh data release are $Y=18.24 \pm 0.03, J=18.04 \pm 0.03, H=+17.68 \pm 0.04$, $K=17.49 \pm 0.06 \mathrm{mag}$, yielding infrared colours $J-H=0.36$ and $H-K=0.19$ mag. We subtracted the infrared contribution of the white dwarf and, using the empirical luminosity-mass relation from Delfosse et al. (2000), we estimated a mass of $0.175 M_{\odot}$ for the secondary star. With the spectral type mass radius relation from Rebassa-Mansergas et al. (2007) this is consistent with a spectral type of M6.5.

SDSS 1434+5335 (SDSS J143443.24+533521.2) has a background or a nearby red object (SDSS J143443.19+533525.0), classified as a galaxy by the SDSS. The background galaxy is too faint, and our object is bright enough that the integration time was relatively short and a possible contamination of our spectra is negligible.

SDSS 1436+5741 and SDSS 2156-0002 present a slight blue excess in the SDSS spectra with respect to single dM stars. Spectroscopic observations carried out for these two systems (see Table 1) showed significant radial velocity variation, confirming their binary nature. We explored GALEX with the aim of learning about the primary stars and found that SDSS 21560002 has GALEX J215614.5-000237 as a counterpart. Only flux in the NUV was detected, with a magnitude of $21.99 \pm 0.34$, consistent with a cold white dwarf.

SDSS 1559+0356 has a hot white dwarf, $T_{\mathrm{eff}}=48212 \mathrm{~K}$, and no companion is visible in the SDSS spectrum. It has a strong $\mathrm{H} \alpha$ emission line from which we were able to measure the radial velocities. SDSS $1559+0356$ was listed as a CV candidate by Szkody et al. (2007), but there is no obvious sign of mass transfer in the spectrum. With its orbital period of $2.2 \mathrm{~h}$, this system is located in the $\mathrm{CV}$ orbital period gap. If it were a detached $\mathrm{CV}$, one would expect a cooler white dwarf (Townsley \& Gänsicke 2009). We investigated infrared surveys to have more information for the secondary star, but no counterpart was found for the object.

SDSS 1611+4640 has the shortest period among the PCEBs, with $P_{\text {orb }}=1.97 \mathrm{~h}$, and it has a cold white dwarf, $T_{\mathrm{eff}} \sim 10300 \mathrm{~K}$ and a late secondary star spectral type, M5.

SDSS 1731+6233 has a red nearby star, and care was taken not to contaminate our spectra with any contribution from it. Unbeknownst to us at the start of our spectroscopic campaign, this system had been previously classified a probable binary by Pourbaix et al. (2005) on the basis of radial velocity variations of $\sim 40 \mathrm{~km} \mathrm{~s}^{-1}$.

SDSS 2149-0717 has SDSS photometry, but it was not an SDSS spectroscopic target. It was identified as a WDMS by Southworth et al. (2007) during some pilot follow-up studies of WDMS candidates in the SDSS footprint at the UK Schmidt telescope with the $6 \mathrm{dF}$ spectrograph. The spectral type of the secondary star is less certain than for SDSS spectra since the $6 \mathrm{dF}$ only covers up to $7500 \AA$.

SDSS 2208+1221 contains the hottest white dwarf in our sample, $T_{\text {eff }} \sim 100000 \mathrm{~K}$ and the earliest spectral type secondary star, a K7 star (Silvestri et al. 2006).

SDSS $2243+3122$ has an M5 \pm 1 so that it could be a detached $\mathrm{CV}(\mathrm{dCV})$ or hibernating $\mathrm{CV}$, which has relaxed to its equilibrium radius. The SDSS spectrum presents the Balmer lines in emission. Radial velocities from the $\mathrm{H} \alpha$ emission line showed no difference in phasing from the NaI absorption doublet; i.e. both sets of lines must originate in the secondary, which argues against a CV nature for the system.

\footnotetext{
$\overline{8}$ http://xcatdb.u-strasbg.fr/
} 
A. Nebot Gómez-Morán et al.: The orbital period distribution of SDSS PCEBs. XII.

Table 1. Log of observations.

\begin{tabular}{|c|c|c|c|c|c|c|c|}
\hline Object & $u$ & $g$ & $r$ & $i$ & $z$ & Spectroscopy: Telescope & \# of spec. \\
\hline SDSS J015225.38-005808.5 & 18.36 & 17.95 & 17.70 & 16.98 & 16.22 & Baade, NTT, WHT & 26 \\
\hline SDSS J022503.02+005456.2 & 21.37 & 20.55 & 19.65 & 18.35 & 17.47 & Baade & 16 \\
\hline SDSS J023804.39-000545.7 & 19.81 & 19.23 & 18.68 & 17.70 & 16.97 & Baade, WHT & 16 \\
\hline SDSS J030138.24 + 050218.9 & 19.11 & 18.39 & 18.05 & 17.27 & 16.61 & VLT, NTT & 21 \\
\hline SDSS J030544.41 - 074941.2 & 18.68 & 18.33 & 18.12 & 17.30 & 16.70 & Baade, Clay, NTT, CA3.5, WHT & 52 \\
\hline SDSS J030716.44 + 384822.8 & 20.64 & 19.04 & 17.86 & 16.70 & 16.06 & CA3.5 & 24 \\
\hline SDSS J032038.72-063822.9 & 19.77 & 19.28 & 19.16 & 18.52 & 17.89 & Baade, NTT, VLT, WHT & 25 \\
\hline SDSS J080736.96 + 072412.0 & 19.44 & 19.13 & 18.84 & 18.05 & 17.46 & VLT, Baade, Clay & 27 \\
\hline SDSS J083354.84 + 070240.1 & 19.15 & 18.73 & 18.75 & 18.15 & 17.60 & VLT, Clay & 19 \\
\hline SDSS J085336.03 + 072033.5 & 19.99 & 19.21 & 18.53 & 17.47 & 16.70 & VLT, Baade & 14 \\
\hline SDSS J092452.39+002449.0 & 18.17 & 18.03 & 18.22 & 17.87 & 17.41 & NTT, VLT & 17 \\
\hline SDSS J094913.37 + 032254.5 & 19.30 & 18.99 & 18.95 & 18.31 & 17.64 & VLT, Clay & 22 \\
\hline SDSS J100609.18 + 004417.0 & 18.32 & 17.85 & 17.70 & 17.36 & 16.68 & Gemini-S, Clay & 14 \\
\hline SDSS J110517.60 + 385125.7 & 19.05 & 18.44 & 18.08 & 17.32 & 16.74 & CA3.5 & 16 \\
\hline SDSS J114312.57 + 000926.5 & 18.43 & 18.15 & 18.17 & 17.60 & 17.03 & Gemini-N, NTT & 13 \\
\hline SDSS J123139.80-031000.3 & 19.41 & 18.67 & 18.19 & 17.09 & 16.26 & NTT, VLT & 18 \\
\hline SDSS J130012.49 + 190857.4 & 19.85 & 19.22 & 18.68 & 17.66 & 16.89 & CA3.5 & 16 \\
\hline SDSS J131334.74 + 023750.7 & 19.12 & 18.67 & 18.53 & 17.73 & 17.05 & VLT, Baade, NTT, WHT & 37 \\
\hline SDSS J131632.04 - 003758.0 & 19.76 & 18.75 & 18.25 & 18.06 & 17.90 & CA3.5 & 10 \\
\hline SDSS J134841.61 + 183410.5 & 17.70 & 17.31 & 17.19 & 16.47 & 15.88 & CA3.5 & 24 \\
\hline SDSS J135228.14 + 091039.0 & 17.85 & 18.13 & 18.56 & 18.81 & 18.83 & Gemini-S, Clay & 17 \\
\hline SDSS J141134.70 + 102839.7 & 18.74 & 18.90 & 19.14 & 19.09 & 18.91 & Gemini-S, Clay & 18 \\
\hline SDSS J142951.19 + 575949.0 & 20.06 & 19.43 & 18.75 & 17.76 & 17.07 & CA3.5, WHT & 21 \\
\hline SDSS J143443.24 + 533521.2 & 15.96 & 15.97 & 16.26 & 16.19 & 15.92 & WHT & 31 \\
\hline SDSS J143642.01 + 574146.3 & 20.87 & 19.73 & 18.60 & 17.38 & 16.69 & CA3.5, WHT & 24 \\
\hline SDSS J143746.69 + 573706.0 & 18.96 & 18.52 & 18.11 & 17.06 & 16.35 & CA3.5 & 10 \\
\hline SDSS J143947.62 - 010606.7 & 16.14 & 16.55 & 16.76 & 16.72 & 16.61 & VLT, Baade, Clay, CA3.5, WHT & 42 \\
\hline SDSS J150657.58 - 012021.7 & 19.61 & 19.09 & 18.94 & 18.26 & 17.67 & Baade, Clay, NTT, WHT, VLT & 17 \\
\hline SDSS J151921.72 + 353625.8 & 16.83 & 16.69 & 17.02 & 17.01 & 16.64 & WHT & 22 \\
\hline SDSS J152359.22 + 460448.9 & 18.23 & 17.92 & 17.76 & 17.18 & 16.40 & WHT & 13 \\
\hline SDSS J152425.21 + 504009.7 & 17.44 & 17.31 & 17.27 & 16.60 & 16.06 & WHT, CA3.5, CA2.2 & 47 \\
\hline SDSS J152821.45 + 344314.9 & 18.45 & 18.43 & 18.46 & 17.86 & 17.28 & WHT, CA3.5 & 19 \\
\hline SDSS J155808.49 + 264225.7 & 19.82 & 19.18 & 18.47 & 17.31 & 16.56 & CA3.5 & 29 \\
\hline SDSS J155904.62 + 035623.4 & 18.23 & 18.40 & 18.58 & 18.68 & 18.68 & Gemini-S, Clay & 18 \\
\hline SDSS J160821.47 + 085149.9 & 19.12 & 18.70 & 18.65 & 18.16 & 17.53 & Gemini-S, Clay & 17 \\
\hline SDSS J161145.88 + 010327.8 & 19.17 & 18.64 & 18.51 & 17.94 & 17.28 & Gemini-N, CA3.5 & 13 \\
\hline SDSS J161113.13 + 464044.2 & 18.28 & 17.72 & 17.84 & 17.48 & 17.02 & WHT & 16 \\
\hline SDSS J162354.45 + 630640.4 & 19.84 & 19.12 & 18.48 & 17.39 & 16.71 & CA3.5, WHT & 29 \\
\hline SDSS J162552.91 + 640024.9 & 19.51 & 19.09 & 18.91 & 18.31 & 17.56 & CA3.5, WHT & 12 \\
\hline SDSS J164615.60 + 422349.2 & 18.73 & 18.41 & 18.54 & 17.95 & 17.22 & WHT & 20 \\
\hline SDSS J170509.65 + 210904.4 & 17.77 & 17.77 & 18.00 & 17.60 & 17.06 & WHT, NTT & 35 \\
\hline SDSS J171810.15 + 610114.0 & 18.28 & 18.00 & 18.20 & 17.78 & 17.19 & WHT & 23 \\
\hline SDSS J173101.49 + 623315.9 & 18.58 & 18.27 & 18.31 & 17.74 & 17.10 & WHT & 14 \\
\hline SDSS J184412.58 + 412029.4 & 17.26 & 16.71 & 16.43 & 15.69 & 14.93 & CA3.5, WHT & 21 \\
\hline SDSS J204541.90 - 050925.7 & 19.04 & 19.04 & 19.06 & 18.51 & 17.99 & VLT, Baade, Clay, NTT, WHT & 34 \\
\hline SDSS J211205.31 + 101427.9 & 18.68 & 18.38 & 18.42 & 18.05 & 17.35 & Gemini-S, WHT & 5 \\
\hline SDSS J211428.41 -010357.2 & 18.71 & 18.64 & 18.47 & 17.83 & 17.24 & Clay, VLT & 26 \\
\hline SDSS J212051.92 - 005827.3 & 18.09 & 17.75 & 17.73 & 17.11 & 16.48 & Clay, WHT & 35 \\
\hline SDSS J212320.74 + 002455.5 & 19.73 & 19.24 & 19.35 & 19.01 & 18.39 & Clay, VLT & 13 \\
\hline SDSS J213218.11 + 003158.8 & 18.40 & 18.03 & 18.16 & 17.89 & 17.49 & Clay, WHT & 17 \\
\hline SDSS J214952.27 - 071756.9 & 17.50 & 16.74 & 16.04 & 15.04 & 14.41 & WHT & 21 \\
\hline SDSS J215614.57 - 000237.4 & 20.77 & 20.14 & 19.55 & 18.28 & 17.31 & Baade, NTT, VLT, WHT & 41 \\
\hline SDSS J220848.99 + 122144.7 & 17.76 & 17.72 & 17.24 & 16.82 & 16.49 & CA3.5, Clay & 19 \\
\hline SDSS J221616.59 + 010205.6 & 18.37 & 17.85 & 17.99 & 17.89 & 17.45 & Gemini-S, WHT & 14 \\
\hline SDSS J224038.37 - 093541.4 & 18.01 & 17.54 & 17.55 & 17.14 & 16.54 & NTT, WHT & 50 \\
\hline SDSS J224307.59 + 312239.1 & 20.76 & 19.59 & 18.69 & 17.28 & 16.26 & CA3.5 & 12 \\
\hline SDSS J231105.66 + 220208.6 & 19.61 & 19.07 & 18.38 & 17.45 & 16.80 & CA3.5 & 29 \\
\hline SDSS J231825.23 - 093539.1 & 18.33 & 18.31 & 18.49 & 18.13 & 17.71 & Baade, NTT, WHT & 39 \\
\hline Object & $\mathrm{u}$ & $\mathrm{g}$ & $\mathrm{r}$ & $\mathrm{i}$ & $\mathrm{Z}$ & Photometry: Telescope & duration, filter \\
\hline SDSS J023804.39-000545.7 & 19.81 & 19.23 & 18.68 & 17.70 & 16.97 & $\mathrm{Ca} 2.2$ & $1.2 \mathrm{~h}, \mathrm{R}$ \\
\hline SDSS J085336.03 + 072033.5 & 19.99 & 19.21 & 18.53 & 17.47 & 16.70 & DuPont & $3.7 \mathrm{~h}, \mathrm{r}$ \\
\hline SDSS J161113.13 + 464044.2 & 18.28 & 17.72 & 17.84 & 17.48 & 17.02 & $\mathrm{Ca} 2.2$ & $5 \mathrm{~h}, \mathrm{R}$ \\
\hline SDSS J211205.31 + 101427.9 & 18.68 & 18.38 & 18.42 & 18.05 & 17.35 & IAC $80, \mathrm{Ca} 2.2$ & $19.3 \mathrm{~h}, \mathrm{I}, \mathrm{R}$ \\
\hline SDSS J213218.11+003158.8 & 18.40 & 18.03 & 18.16 & 17.89 & 17.49 & Ca2.2 & 4.5 h, R \\
\hline SDSS J224038.37 -093541.4 & 18.01 & 17.54 & 17.55 & 17.14 & 16.54 & $\mathrm{Ca} 2.2$ & $3.5 \mathrm{~h}, \mathrm{R}$ \\
\hline SDSS J224307.59 + 312239.1 & 20.76 & 19.59 & 18.69 & 17.28 & 16.26 & IAC80 & $26.47 \mathrm{~h}, \mathrm{i}$ \\
\hline
\end{tabular}




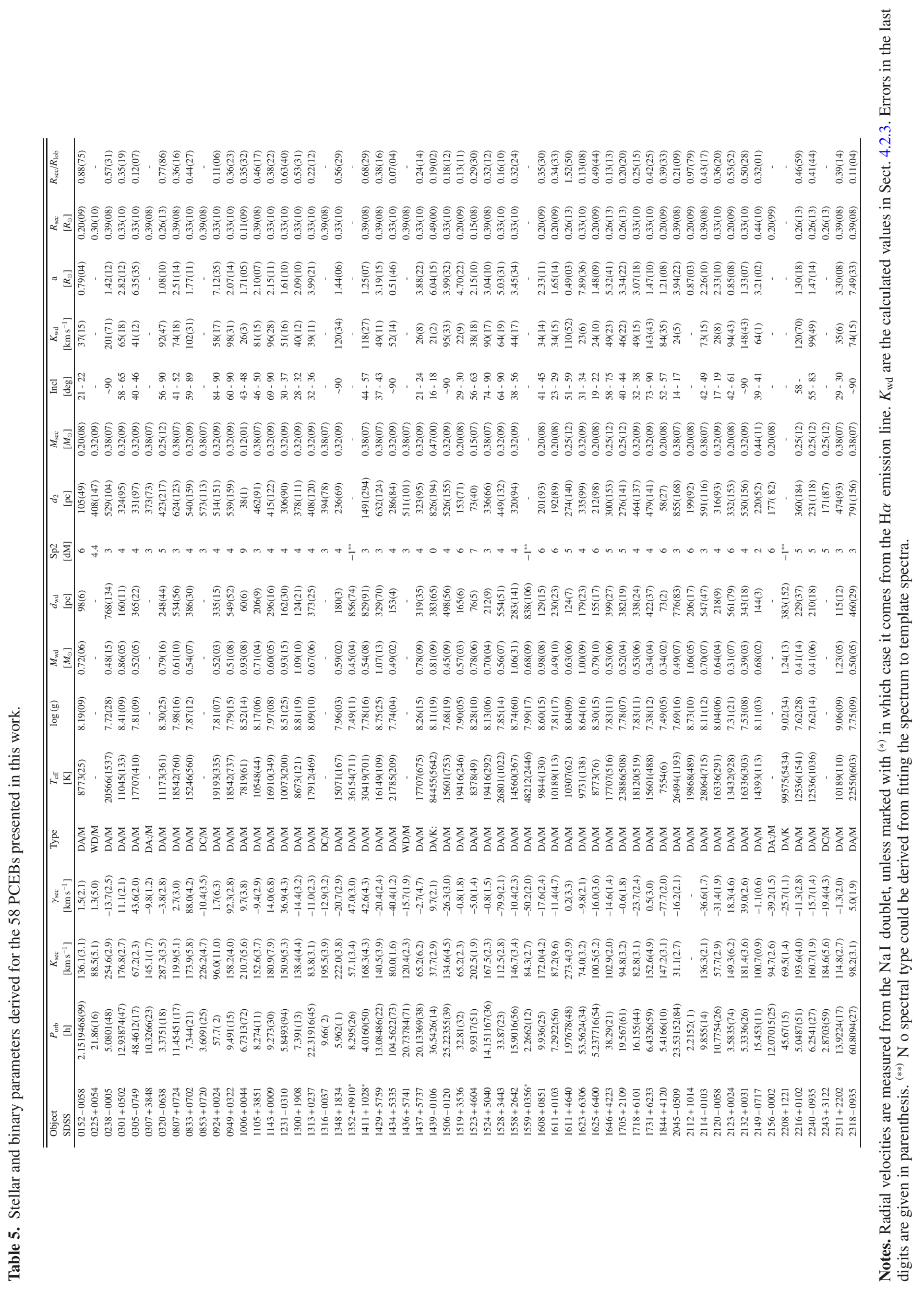

A43, page 16 of 20 
A. Nebot Gómez-Morán et al.: The orbital period distribution of SDSS PCEBs. XII.
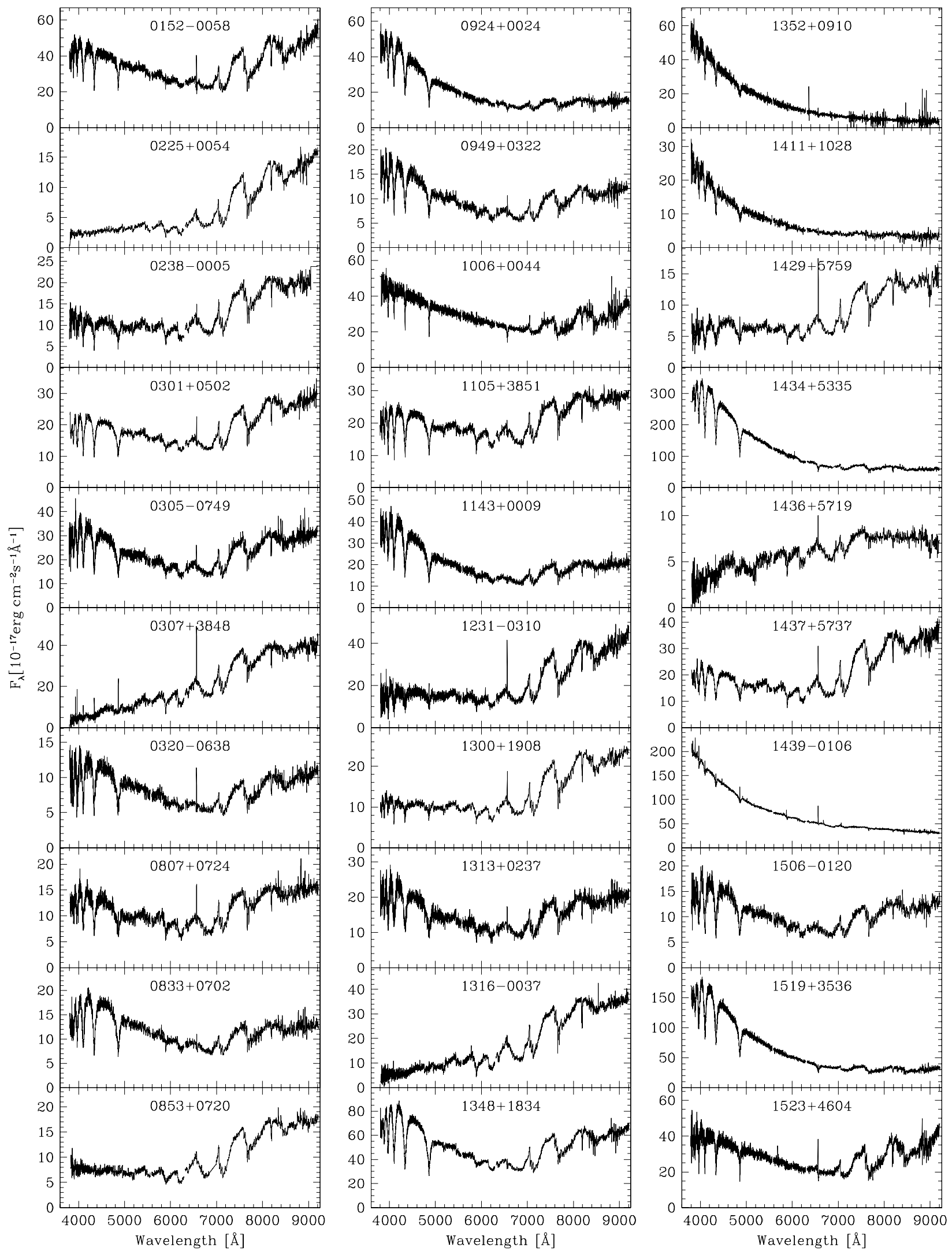

Fig. 3. SDSS spectra of the 58 systems with orbital period measured in this paper. Systems are sorted in right ascension from left to right and from top to bottom. 

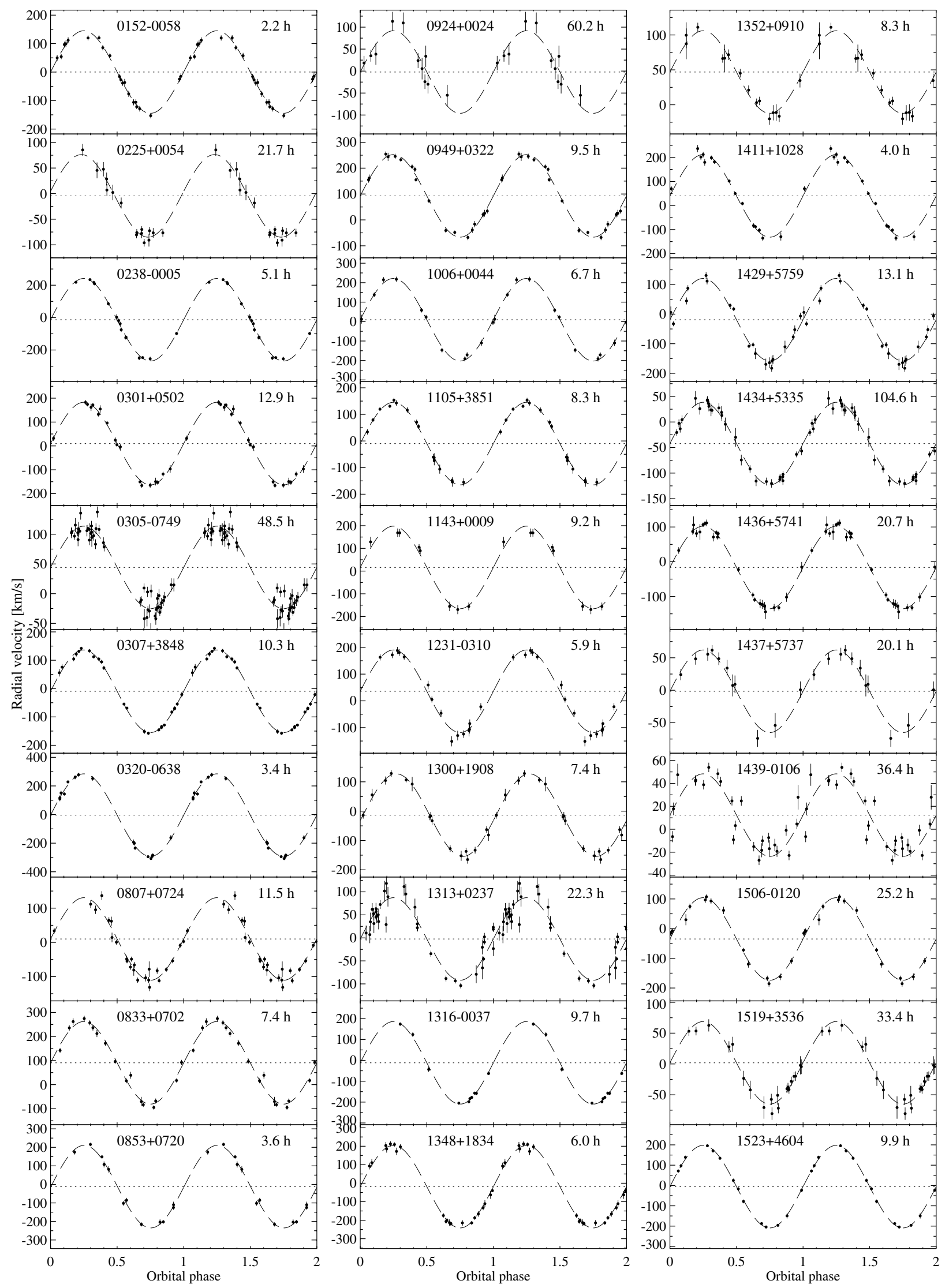

Fig. 4. Phase-folded radial velocities curves and sine fits to the data (dashed lines). As for Fig. 3, systems are sorted in right ascension from left to right and from top to bottom. 
A. Nebot Gómez-Morán et al.: The orbital period distribution of SDSS PCEBs. XII.
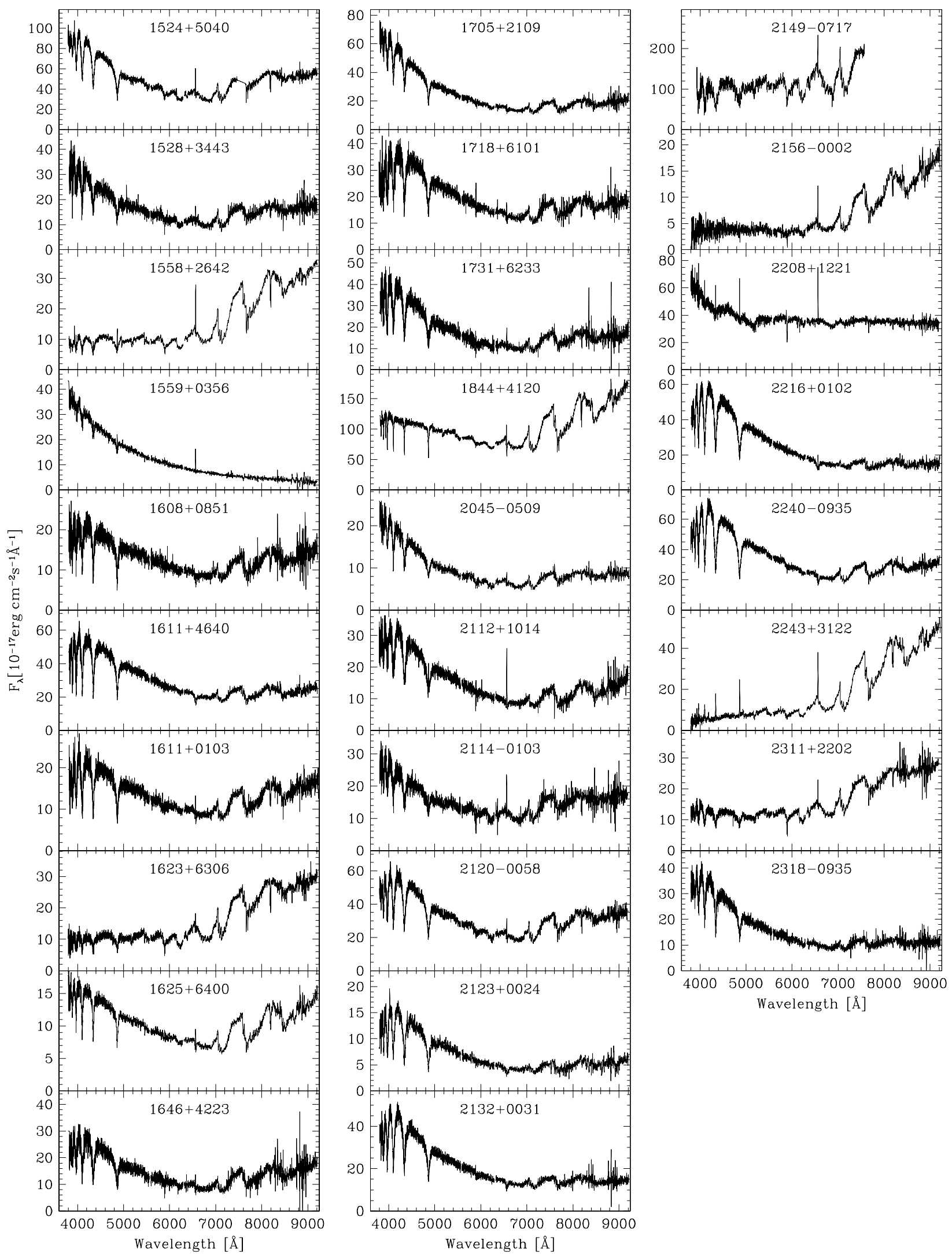

Fig. 5. SDSS spectra of the 58 systems with orbital period measured in this paper. Systems are sorted in right ascension from left to right and from top to bottom. 

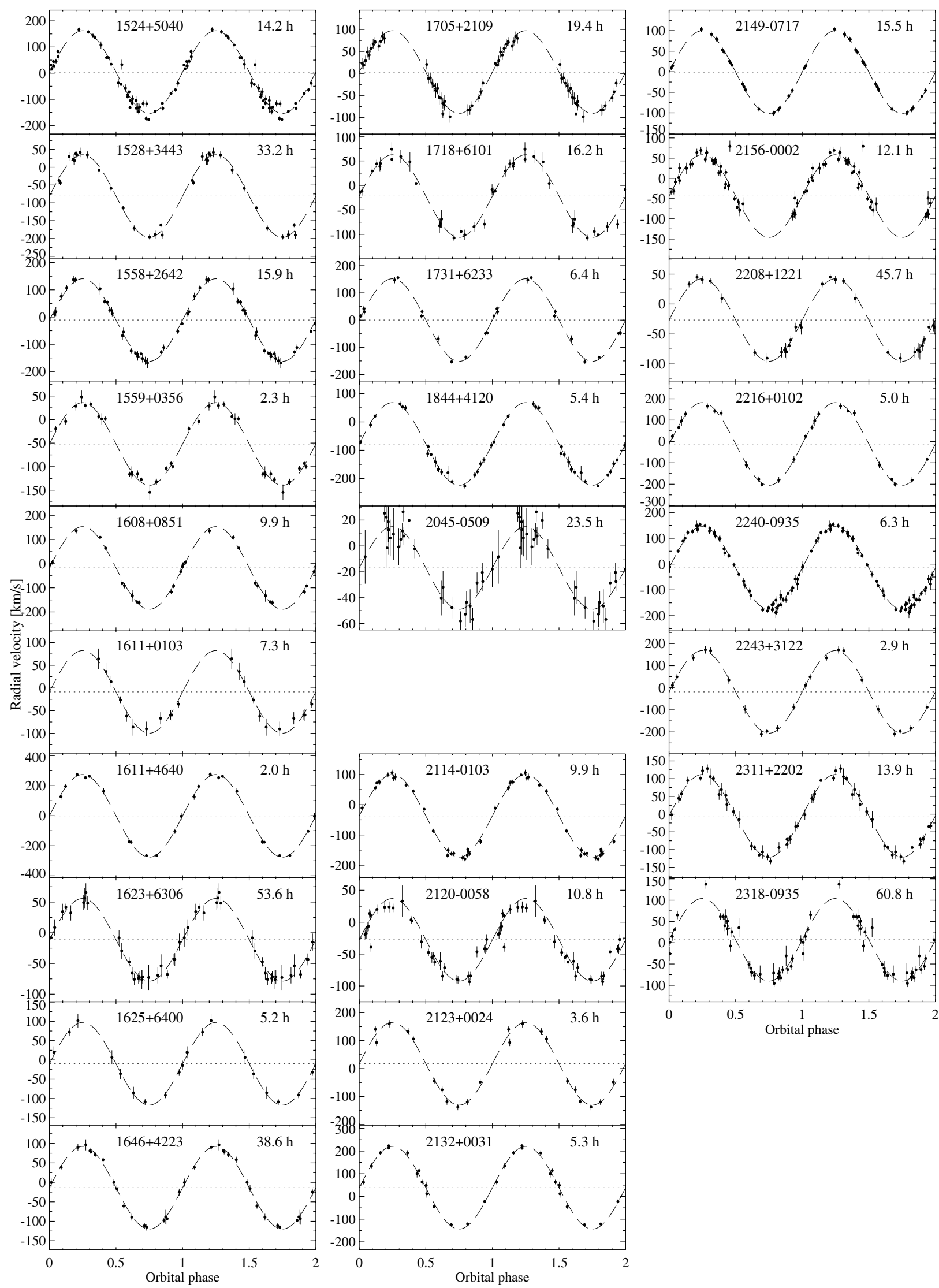

Fig. 6. Phase-folded radial velocities curves and sine fits to the data (dashed lines). As for Fig. 3, systems are sorted in right ascension from left to right and from top to bottom. 